Research Article

\title{
Sensitivity Study on the Influence of Parameterization Schemes in WRF_ARW Model on Short- and Medium-Range Precipitation Forecasts in the Central Andes of Peru
}

\author{
Aldo S. Moya-Álvarez $\mathbb{D}^{D},{ }^{1}$ Daniel Martínez-Castro, ${ }^{1,2}$ José L. Flores, ${ }^{1}$ and Yamina Silva ${ }^{1}$ \\ ${ }^{1}$ Instituto Geofísico del Perú, Lima, Peru \\ ${ }^{2}$ Instituto de Meteorología de Cuba, La Habana, Cuba
}

Correspondence should be addressed to Aldo S. Moya-Álvarez; amoya@igp.gob.pe

Received 15 February 2018; Revised 14 April 2018; Accepted 29 April 2018; Published 22 May 2018

Academic Editor: Mario M. Miglietta

Copyright ( 2018 Aldo S. Moya-Álvarez et al. This is an open access article distributed under the Creative Commons Attribution License, which permits unrestricted use, distribution, and reproduction in any medium, provided the original work is properly cited.

A sensitivity study of the performance of the Weather Research and Forecasting regional model (WRF, version 3.7) to the use of different microphysics, cumulus, and boundary layer parameterizations for short- and medium-term precipitation forecast is conducted in the Central Andes of Peru. Lin-Purdué, Thompson, and Morrison microphysics schemes were tested, as well as the Grell-Freitas, Grell 3d, and Betts-Miller-Janjic cumulus parameterizations. The tested boundary layer schemes were the Yonsei University and Mellor-Yamada-Janjic. A control configuration was defined, using the Thompson, Grell-Freitas, and Yonsei University schemes, and a set of numerical experiments is made, using different combinations of parameterizations. Data from 19 local meteorological stations and regional and global gridded were used for verification. It was concluded that all the configurations overestimate precipitation, but the one using the Morrison microphysical scheme had the best performance, based on the indicators of bias $(B)$ and root mean square error (RMSE). It is recommended not to use the Betts-Miller-Janjic scheme in this region for low resolution domains. Categorical forecast verification of the occurrence of rainfall as a binary variable showed detection rates higher than $85 \%$. According to this criterion, the best performing configuration was the combination of Betts-Miller-Janjic and Morrison. Spatial verification showed that, even if all the configurations overestimated precipitation in some degree, spatial patterns of rainfall match the TRMM and PISCO rainfall data. Morrison's microphysics scheme shows the best results, and consequently, this configuration is recommended for short- and medium-term rainfall forecasting tasks in the Central Andes of Peru and particularly in the Mantaro basin. The results of a special sensitivity experiment showed that the activation or not of cumulus parametrization for the domain of $3 \mathrm{~km}$ resolution is not relevant for the precipitation forecast in the study region.

\section{Introduction}

Mesoscale meteorological models are a powerful tool, both for operational simulation and for atmospheric investigations [1]. In this sense, the Weather Research and Forecasting (WRF) model [2] is currently one of the most used in the world for these purposes, since it supports the use of very high-resolution grids for domains in any region of the planet and allows changes in its physics schemes configuration to tune it for regional conditions without having to compile it every time. Its development began in the second half of the 1990s as a collaborative partnership, mainly between the National Center for Atmospheric Research (NCAR), the National Oceanic and Atmospheric Administration (NOAA), the Air Force Time Agency (AFWA), the Naval Research Laboratory, the University of Oklahoma, and the Federal Aviation Administration (FAA), all of the United States of America.

In the present work, a research is carried out to evaluate how the WRF model simulates rainfall in the Peruvian Central Andes. For validation, observational data from 19 stations in the region are used, as well as gridded observational 
data. Most of the rainfall in Peru is concentrated in the period between the months of September and April [3], defining a marked seasonality, with a dry season between May and August [4-6]. In this region, rainfall plays an important economic role, since $71 \%$ of the arable land in the basin depends on it for crops [7].

Regional weather numerical models, including WRF, have been previously used to carry out studies on different mountainous regions, including part of the Andes [8-11]. However, in Peruvian Central Andes, numerical modeling studies are scarce. In a recent paper [12], the influence of orography on the diurnal cycle of rainfall in the Central Andes has been investigated using WRF. However, there are no studies evaluating the sensitivity of the model to changes in its configuration; for example, the use of certain parameterization schemes of physical processes or the characteristics of the domains, which is of great interest due to the orographic complexity of the region, may be relevant to the forecasting skill of the model for operational applications. In the present investigation, WRF sensitivity to changes in its cumulus parameterization, microphysics, and boundary layer schemes is evaluated, relative to the results of its short- and medium-term precipitation forecast in the study region.

In the specialized bibliography, as well as in the user manual, there are dissimilar parametrization schemes of the different physical processes that WRF solves, among them those of boundary layer, clusters, and microphysics, which would be the objects of verification of this work. Thus, among others, for the boundary layer, there are the Mellor-Yamada-Janjic scheme (MYJ) [13] and the Yonsei University scheme (YSU) [14]. For convection, the parameterizations of Betts-Miller-Janjic (BMJ) [15], Grell 3D (GRELL3), and Grell-Freitas [16], were used, and for microphysics, the parameterizations of Thompson [17], Morrison [18], and Lin-Purdué [19] were used.

High-resolution numerical modeling has specific issues to deal with, as the choice to include or not cumulus parameterization schemes (CPS), and explicit microphysics. The WRF user manual (v. 3.7) suggests the explicit solution of convection for grids with these resolutions; however, in the scientific literature, there does not seem to be a total consensus on this aspect. For example, in [20], it is proposed that an explicit treatment of convection for a resolution, for example, of $4 \mathrm{~km}$, shows a more accurate description of the physics of convective systems but not necessarily provides a better forecast. Thus, in [21], although it is stated that "Idealized modeling demonstrates that, at a fine resolution such as 2 or $4 \mathrm{~km}$, classic super-cellular convective features including a reflectivity hook, midlevel rotation, and storm splitting can be resolved explicitly, and CPS is not needed," but it is concluded that it is not appropriate to assume that a simulation that uses a grid of small spacing $(<5 \mathrm{~km})$ will not need a CPS. This will depend on the synoptic forcing and the time of the year.

Consequently, and bearing in mind that the objective of this research is to verify the sensitivity of WRF to the use of different parametrization schemes for punctual precipitation forecast in this complex region, it was decided to include the convection parameterization schemes also for the domain of $3 \mathrm{~km}$ resolution. However, a special numerical experiment was made, using the best performing configuration, deactivating the cumulus parameterization for the domain of $3 \mathrm{~km}$ resolution to investigate the sensitivity of the forecast to this procedure.

\section{Data and Methodology}

2.1. Initial and Border Conditions. The experiments were carried out using model WRF v. 3.7 (Weather Research and Forecasting, version 3.7). The initial and boundary conditions were taken from the "Global Operational Analysis" of the National Center of Environmental Prediction (NCEP), final analysis FNL (https://rda.ucar.edu/datasets/ds083.2/), every 6 hours, with horizontal resolution of $1^{\circ} \times 1^{\circ}$. The analysis contains surface data and 26 higher air levels and includes the following surface variables: sea level pressure, higher air variables (geopotential height, air temperature, and zonal and meridional components of the wind and vertical air velocity), sea surface temperature, and soil parameters. Even though there are currently Global Forecast System (GFS) outputs with resolution of $0.5^{\circ}$ and $0.25^{\circ}$ available, in the present research, border conditions of FNL with coarser resolution were used, with the objective that the physical WRF parameterizations determine the behavior of the forecast in the largest possible measure. On the contrary, for a sensitivity study, analysis data are preferred over prognostic output, which would have introduced implicit additional forecast uncertainty.

2.2. Topography Data. The topographic data Global 30 ArcSecond Elevation (GTOPO30) of the Geological Survey of the United States (USGS), which is a default option in the model, were replaced by the digital elevation model of the Shuttle Radar Topography Mission (SRTM; https://dds.cr. usgs.gov/srtm/version2_1/) [22, 23], with a resolution of $90 \mathrm{~m}$, which for South America has an average horizontal error of $9.0 \mathrm{~m}$ and an average absolute vertical error of $6.2 \mathrm{~m}$ and improves about 10 times (both in spatial resolution and in vertical accuracy) the continental digital elevation model GTOPO30 [24].

2.3. Model Configuration. The simulations are performed for three domains (Figure 1), whose characteristics are specified in Table 1. The unidirectional nesting technique has been applied in the simulations, running all three domains at the same time. The model is initialized at 12 UTC in all cases.

The model was configured with 28 vertical levels, up to approximately 16,000 meters, for which the variable "e_vert" was used in the configuration of the "namelist.input." In this case, $57 \%$ of the levels were framed below 6000 meters.

A "control" simulation was defined, and the cumulus, boundary layer, and microphysical schemes were subsequently changed in the rest of the numerical experiments (only one at a time).

To carry out the sensitivity study, we have previously considered previous work results in similar conditions, 

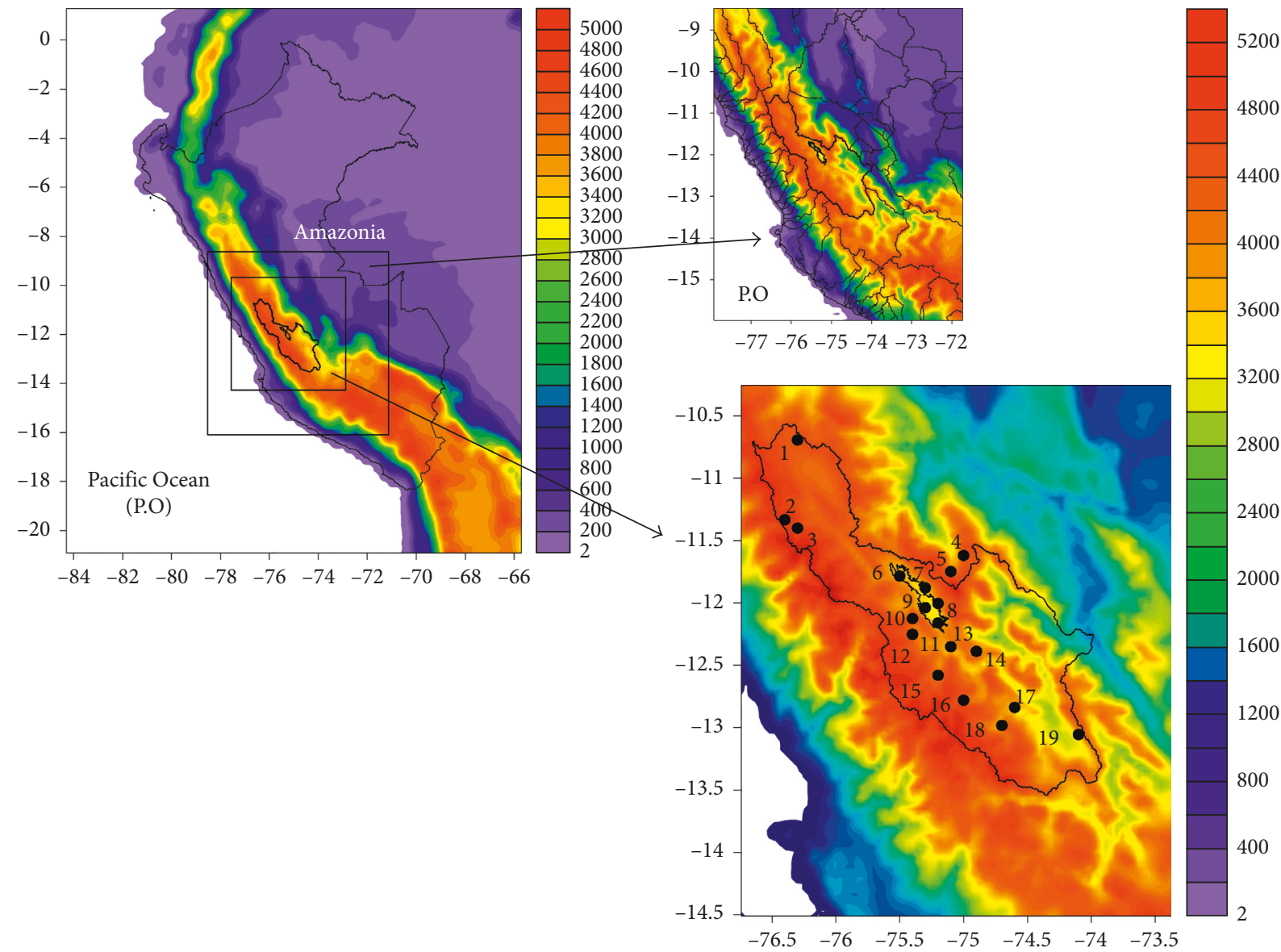

Figure 1: Domains used in the simulations and distribution of the stations considered for the verification of the results.

TABLE 1: Main characteristics of domains, initial, and boundary conditions.

\begin{tabular}{lccc}
\hline Characteristics & Domain 1 & Domain 2 & Domain 3 \\
\hline Central point & Latitude: $10^{\circ} \mathrm{S}$ & Latitude: $12.25819^{\circ} \mathrm{S}$ & Latitude: $12.39203^{\circ} \mathrm{S}$ \\
Horizontal grid step & Longitude: $75^{\circ} \mathrm{S}$ & Longitude: $74.8356^{\circ} \mathrm{S}$ & Longitude: $75.0548^{\circ} \mathrm{S}$ \\
$\begin{array}{l}\text { Dimension (XYZ) } \\
\text { Time step }\end{array}$ & $18 \mathrm{~km}$ & $6 \mathrm{~km}$ & $3 \mathrm{~km}$ \\
Initial and contour conditions & $90 \mathrm{~s}$ & $115 \times 142 \times 28$ & $125 \times 161 \times 28$ \\
& FNL $1.0^{\circ} \times 1.0^{\circ}$ & $36 \mathrm{~s}$ & $18 \mathrm{~s}$ \\
\end{tabular}

TABle 2: Parameterization schemes used in the experiments.

\begin{tabular}{|c|c|c|c|c|c|c|}
\hline \multirow{2}{*}{ Parameters } & \multicolumn{6}{|c|}{ Experiments } \\
\hline & CTR & C_BMJ & C_GRELL3 & MP_MR & MP_LP & BL_MYJ \\
\hline Microphysics & Thomson & Thomson & Thomson & Morrison & Lin et al. & Thomson \\
\hline Cumulus & Grell-Freitas & Betts-Miller-Janjic & Grell 3D & Grell-Freitas & Grell-Freitas & Grell-Freitas \\
\hline Boundary layer & Yonsei university & Yonsei university & Yonsei university & Yonsei university & Yonsei university & Mellor-Yamada-Janjic \\
\hline Radiation LW & RRTMG & RRTMG & RRTMG & RRTMG & RRTMG & RRTMG \\
\hline Radiation SW & RRTMG & RRTMG & RRTMG & RRTMG & RRTMG & RRTMG \\
\hline
\end{tabular}

focusing on orographic complexity and/or tropical condition. Thus, the parametrization schemes used in the control simulation (CTR in Table 2) and in the rest of experiments were mostly based on the suggestions of the WRF v. 3.7 "User Guide" and in previous results of sensitivity studies and applications of the model in the Andes [12] and in domains with complex topography in tropical and subtropical regions, such as the Himalayas $[25,26]$, the south of India [27], and the Caribbean [28].

For the control experiment, the following parameterization schemes were selected: for microphysics, the Thompson scheme, the soil model is the so-called Unified 
Noah Land Surface Model [29]; for convection, the GrellFreitas parameterization, it explicitly represents updraft and downdraft and includes the "detrainment" of cloud and ice.

For the boundary layer, the YSU scheme was used, and for the surface layer, the MM5 similarity scheme was used [30-34].

The radiation model was the RRTMG (rapid radiative transfer model for general circulations models) [35], which is a relatively recent version of the RRTM (rapid radiative transfer model) [36], with a better representation of cloudiness, unresolved by the mesh of the model. It applies the $k$-correlated method to implement an algorithm that is characterized by its speed [37].

Six configurations of the model were tested, which have been summarized in Table 2: the control configuration (CTR); C_BMJ and C_GRELL3, with Betts-Miller-Janjic and Grell 3D as cumulus parameterizations; MP_MR and MP_LP, where Morrison and Lin-Purdué parameterizations have been used cumulus; and BL_MYJ, where the Mellor-Yamada-Janjic boundary layer scheme was applied. Notice that the acronyms of the configurations are composed by the type of the scheme that substitutes the one in the control configuration, CTR ( $\mathrm{C}$, convection; MP, microphysics; BL, boundary layer), and two or three letters referring to the applied scheme.

2.4. Study Period and Verification of Results. The simulations were carried out with a forecast horizon of up to 10 days, for which 9 tens (periods of ten consecutive days) of the months of December, January, and February of the years 2007, 2009, 2010, 2011, and 2012 were selected. All the selected dates belong to the rainy period of the year. The particular tens were chosen among the rainiest for each month. Figure 2 shows the average of 24-hour rainfall for each forecast period for the 19 weather stations considered for punctual verification during the 9 selected tens.

The stations considered belong to the observation network of the National Meteorology and Hydrology Service of Peru (SENAMHI), whose data are subject to verification.

The tens considered were the following:

(1) First ten of January 2007

(2) Second ten of January 2007

(3) Second ten of February 2007

(4) Third ten of December 2007

(5) Second ten of February 2009

(6) First and ten of February 2010

(7) Third ten of February 2010

(8) Third ten of January 2011

(9) First ten of February 2012.

The coordinates and altitude of the stations considered for the study are shown in Table 3 .

The verification of the results is carried out in three ways: punctual verification the forecast of 24-hour precipitation values, using numerical descriptive statistical measures, the second, punctual verification of the occurrence of

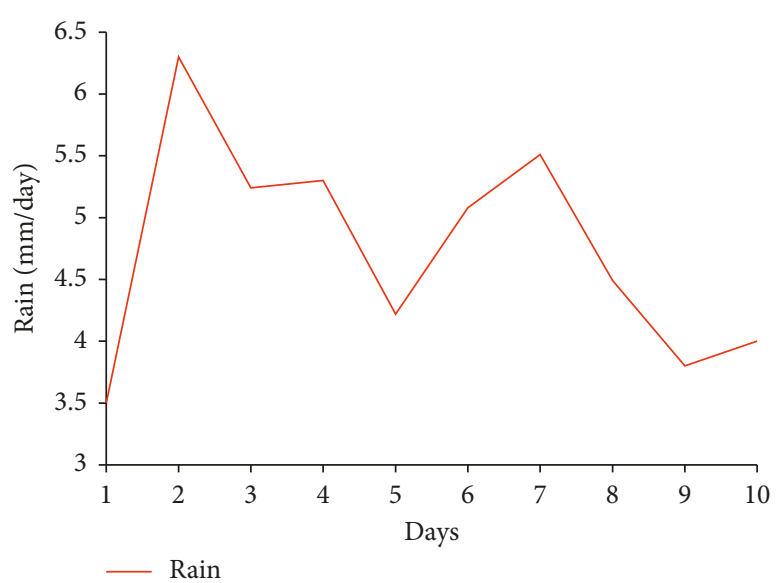

Figure 2: Mean of accumulated 24-hour precipitation for each forecast period in the 19 meteorological stations considered during the 9 selected tens.

precipitation as a categorical binary variable, and the third, verification of the ability of the model to reproduce patterns of spatial distribution of precipitation. Model output was interpolated using the Cressman method [38] to the stations grid points and compared to the stations grid point with the "in situ" measured data on the basis of the following statistics: bias (B), root mean squared error (RMSE), and mean absolute error (MAE) are calculated as follows:

$$
\begin{aligned}
B_{k} & =\sum_{i=1}^{N_{\mathrm{s}}} \sum_{j=1}^{N_{\mathrm{p}}} \frac{\mathrm{Pf}_{i j}-\mathrm{Po}_{i j}}{N}, \\
\mathrm{RMSE}_{k} & =\sqrt{\sum_{i=1}^{N_{\mathrm{s}}} \sum_{j=1}^{N_{\mathrm{p}}}\left(\frac{\mathrm{Pf}_{i j}-\mathrm{Po}_{i j}}{N}\right)^{2}}, \\
\mathrm{MAE}_{k} & =\sum_{i=1}^{N_{\mathrm{s}}} \sum_{j=1}^{N_{\mathrm{p}}}\left|\frac{\mathrm{Pf}_{i j}-\mathrm{Po}_{i j} \mid}{N}\right|,
\end{aligned}
$$

where $B_{k}, \mathrm{RMSE}_{k}$, and $\mathrm{MAE}_{k}$ are the bias, root mean square error, and absolute mean error for the $k$ forecast term $(k=1,10)$, respectively. $\mathrm{Pf}_{i j}$ and $\mathrm{Po}_{i j}$ are the simulated (forecast) and observed precipitation values, respectively, for the $i$ th station and the $j$ th period of ten days $(i=1,19$; $j=1,9)$.

" $B$ " estimates the difference of the mean value of the numerical forecast relative to the observations; "RMSE" is a measure of the magnitude of the mean error of the simulation with respect to the observations, while "MAE" constitutes the mean absolute deviation of the simulated variable with respect to the observations.

The binary categorical verification was carried out with the help of the so-called "contingency table," used in [39], but in our case, the fuzzy verification technique was not used, but direct point-to-point verification was used, which is based on the point-to-point comparison of the predicted and observed fields and the calculation of several indices, such as the probability of detection (POD), the proportion of 
TABLE 3: Stations used to validate the simulations.

\begin{tabular}{|c|c|c|c|c|c|c|}
\hline Number & Name & Abbreviation & Longitude & Latitude & Altitude $(\mathrm{m})$ & Location \\
\hline 1 & Cerro de Pasco & CER & -76.3 & -10.693 & 4260 & North \\
\hline 2 & Yantac & YAN & -76.4 & -11.333 & 4600 & North \\
\hline 3 & Marcapomacocha & MAR & -76.3 & -11.4 & 4413 & North \\
\hline 4 & Ricran & RIC & -75 & -11.619 & 3500 & Center \\
\hline 5 & Comas & $\mathrm{COM}$ & -75.1 & -11.748 & 3300 & Center \\
\hline 6 & Jauja & JAU & -75.5 & -11.784 & 3322 & Center \\
\hline 7 & Ingenio & ING & -75.3 & -11.879 & 3450 & Center \\
\hline 8 & Santa Ana & STA & -75.2 & -12.004 & 3295 & Center \\
\hline 9 & Huayao & HYO & -75.3 & -12.038 & 3308 & Center \\
\hline 10 & S. J. de Jarpa & JAR & -75.4 & -12.125 & 3726 & Center \\
\hline 11 & Vieques & VIQ & -75.2 & -12.16 & 3186 & Center \\
\hline 12 & Laive & LAI & -75.4 & -12.252 & 3990 & Center \\
\hline 13 & Pilchaca & PIL & -75.1 & -12.35 & 3570 & Center \\
\hline 14 & Pampas & PAM & -74.9 & -12.388 & 3260 & Center \\
\hline 15 & Huancapi & $\mathrm{HCP}$ & -75.2 & -12.58 & 3800 & South \\
\hline 16 & Huancavelica & $\mathrm{HCV}$ & -75 & -12.78 & 3676 & South \\
\hline 17 & Acobamba & $\mathrm{ACO}$ & -74.6 & -12.838 & 3236 & South \\
\hline 18 & Lircay & LIR & -74.7 & -12.982 & 3150 & South \\
\hline 19 & La Quinua & QUI & -74.1 & -13.055 & 3260 & South \\
\hline
\end{tabular}

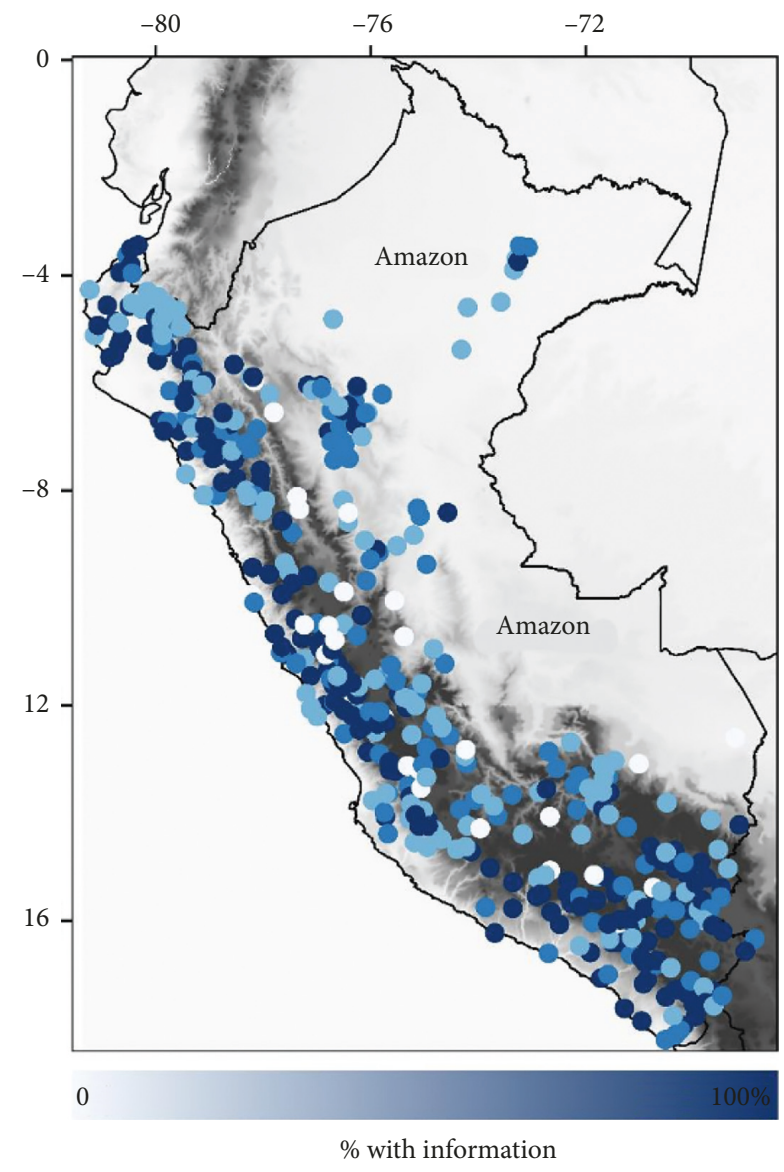

FIGURE 3: Network of stations used for the elaboration of PISCO (taken from [42]), with percentages of data per station, considering the period 1981-2016.

false alarms (FAR), the bias $(B)$, and the "negative correct" (NC). As a criterion of the precipitation event, a $0.5 \mathrm{~mm}$ threshold was used.
In this case, a modification to the ACC (accuracy) index is proposed, and the Weighted Effectiveness Index (WEI) is introduced (2), which increases the relative weight of the misses, based on the criterion that a precipitation event that has occurred without being predicted, will have a greater negative impact on society and the economy than a false alarm:

WEI $=\frac{\text { hits }+ \text { negative corrects }}{\text { hits }+ \text { negative corrects }+ \text { falses alarms }+1.25 * \text { misses }}$,

where hits = the event was forecast and it happened; false alarms = the event was forecast and it did not happen; negative corrects $=$ the event was not forecast and it did not happen; and misses = the event was not forecast and it happened.

The spatial verification was carried out using the gridded data of the product 3B42 of the "Tropical Rainfall Measuring Mission" (TRMM 3B42), which is composed of a set of global "multisatellite" precipitation analysis data with a horizontal resolution of $0.25^{\circ}$ and time resolution of $3 \mathrm{~h}$, available between $50^{\circ} \mathrm{S}$ and $50^{\circ} \mathrm{N}[40,41]$ and of the "Peruvian Interpolate data of the SENAMHI's Climatological and Hydrological Observations" (PISCO) [42]. In this sense, it is important to point out that PISCO data have little information in the area of the Peruvian Amazonia (Figure 3), which could influence the generated precipitation patterns [42]. To obtain the spatial distribution of bias, the TRMM grid was adjusted (by interpolation) to the output grid of WRF and subsequently the difference of the predicted grid and that of TRMM was calculated.

\section{Results and Discussion}

3.1. Results of Punctual Verification: 24-Hour Cumulative Precipitation. Table 4 shows the results of the simulations in each domain in terms of forecast quality measures. 
TABLE 4: Statistics of the point verification of precipitation forecast for the 3 simulated domains and 6 combinations of parametrizations, including the control experiment, average of all the stations considered.

\begin{tabular}{|c|c|c|c|c|c|c|c|}
\hline \multirow{2}{*}{ WRF_DOM } & \multirow{2}{*}{ Statistics } & \multicolumn{6}{|c|}{ Parametrizations } \\
\hline & & CTR & C_BMJ & C_GRELL3 & MP_MR & MP_LP & BL_MYJ \\
\hline \multirow{3}{*}{ 1: $18 \mathrm{~km}$} & B (mm/day) & 7.35 & 12.08 & 6.63 & 6.82 & 7.97 & 8.73 \\
\hline & RMSE (mm/day) & 13.59 & 17.08 & 12.41 & 12.49 & 14.27 & 14.03 \\
\hline & MAE (mm/day) & 10.17 & 13.84 & 9.37 & 9.63 & 10.62 & 11.18 \\
\hline \multirow{3}{*}{$2: 6 \mathrm{~km}$} & $\mathrm{~B}(\mathrm{~mm} /$ day $)$ & 6.01 & 6.71 & 5.78 & 4.66 & 6.06 & 6.19 \\
\hline & RMSE (mm/day) & 12.16 & 11.67 & 11.76 & 10.62 & 12.35 & 12.28 \\
\hline & MAE (mm/day) & 9.09 & 9.23 & 8.92 & 8.07 & 9.18 & 9.31 \\
\hline \multirow{3}{*}{ 3: $3 \mathrm{~km}$} & $\mathrm{~B}(\mathrm{~mm} /$ day $)$ & 5.31 & 5.71 & 5.46 & 3.68 & 4.87 & 5.98 \\
\hline & RMSE (mm/day) & 11.69 & 10.21 & 11.3 & 10.02 & 11.68 & 12.17 \\
\hline & MAE (mm/day) & 9.7 & 8.08 & 8.52 & 7.32 & 8.45 & 9.19 \\
\hline
\end{tabular}

The smaller errors are given in bold.

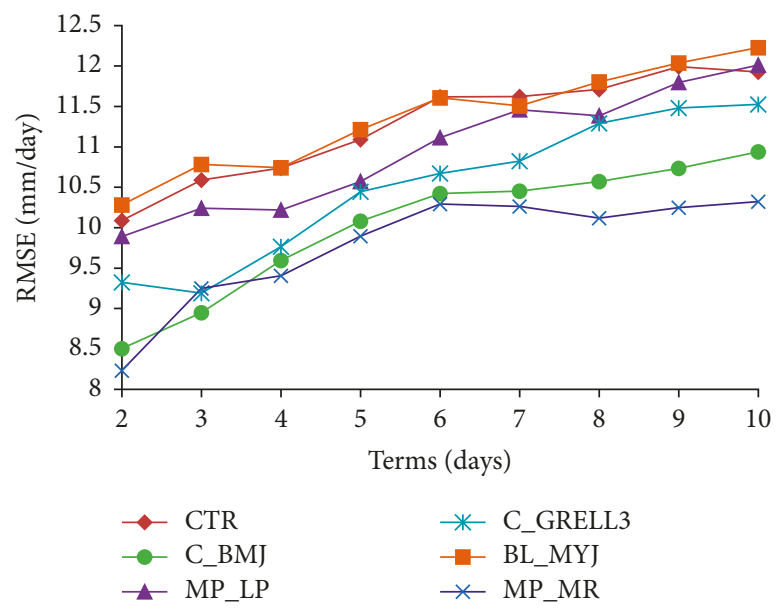

Figure 4: Evolution of the "RMSE" average of domains 2 and 3 of all stations with each configuration scheme used, for the 10 days of simulation horizon.

It can be seen that the model overestimated precipitation in all domains regardless of the configuration. This is consistent with [12], who found that WRF shows a significant positive bias over the Tropical Andes, when comparing the results of the diurnal cycle produced by the model with reference to the TRMM 3B42 product.

For the $18 \mathrm{~km}$ domain, the best results were obtained with the C_GRELL3 scheme $(6.63 \mathrm{~mm} /$ day $)$, which at the same time showed an "RMSE" of $12.41 \mathrm{~mm} /$ day and an "MAE" of $9.37 \mathrm{~mm} /$ day. The poorer result was obtained when the configuration C_BMJ was applied, which showed a significantly higher " $B$ " than the rest of the experiments $(12.08 \mathrm{~mm} /$ day $)$.

For the $6 \mathrm{~km}$ domain, the MP_MR configuration gave the best results, with a bias of 4.66, "RMSE" of 10.62, and "MAE" of $8.07 \mathrm{~mm} /$ day. For this domain, all the configurations showed a remarkable improvement of their indicators in relation to the domain of $18 \mathrm{~km}$. In the particular case of C_BMJ, it showed bias of 6.71 and "RMSE" of 11.67, very similar to the rest of the configurations, so it can be partially concluded that this configuration can be used for high-resolution domains, for example, $6 \mathrm{~km}$, but not for lowresolution domains, this is $18 \mathrm{~km}$ in this case.

For the $3 \mathrm{~km}$ domain, the MP_MR configuration also showed the best indicators with bias of 3.68, "RMSE" of
10.02, and "MAE" of 7.32. The verification indicators did not show significant improvement for this domain in relation to those achieved in the $6 \mathrm{~km}$ domain.

So in general, the MP_MR configuration gave the best results, mainly for the finer domains.

Figure 4 shows the evolution of the average "RMSE" for the 10 days of the simulation horizon, between domains 2 and 3 with each configuration used. It is noted that the "RMSE" increases as the simulation period increases. The configuration C_BMJ and MP_MR shows relatively low RMSE values in all forecast days, growing slowly from day 1 to day 5 , but from the sixth day of forecast on, C_BMJ continues increasing the error, while MP_MR curve shows almost no slope until day 10. Here, the MP_MR scheme showed the best behavior regarding RMSE during the whole period.

Figure 5 shows similar results as Figure 4, but for the $B$ indicator. As can be seen, the lowest " $B$ " was shown by the MP_MR configuration, producing the less rainfall overestimation. Figures 4 and 5 indicate that the MP_MR was not only the configuration showing the best results in a general way but also had the most stable behavior throughout the forecast horizon. Based on the point verification, it is further shown that all the tested configurations overestimate precipitation in the study area. 


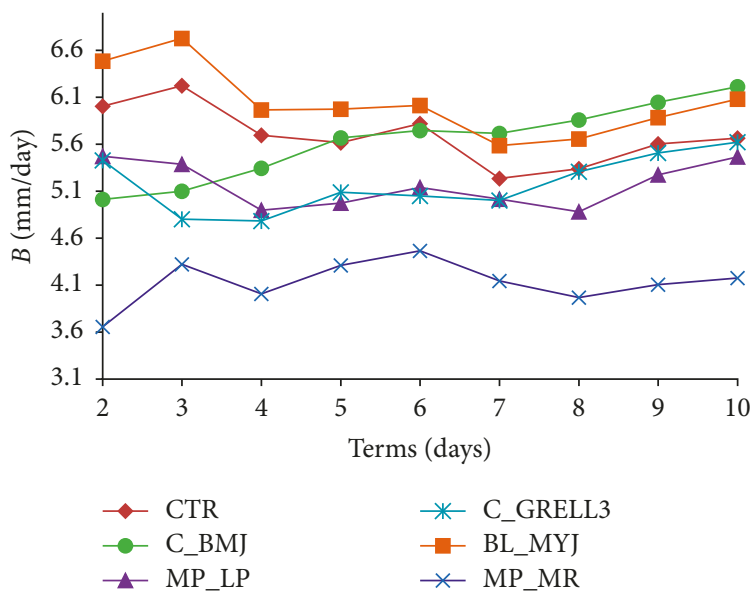

FIgURE 5: Evolution of the average " $B$ " of domains 2 and 3 with each configuration, for the 10 days of the simulation horizon.

TABLE 5: Statistics of the point verification for the domains 2, 3, and 6 combinations of parametrizations, including the control experiment, average of all the stations considered.

\begin{tabular}{lccccccc}
\hline \multirow{2}{*}{ WRF_DOM } & \multirow{2}{*}{ Statistics } & CTR & C_BMJ & C_GRELL3 & MP_MR & MP_LP & BL_MYJ \\
\hline \multirow{3}{*}{$2: 6 \mathrm{~km}$} & POD (\%) & 81.54 & $\mathbf{9 4 . 3 9}$ & 90.61 & 94.04 & 92.28 \\
& FAR (\%) & 28.40 & 28.55 & 29.00 & 29.10 & $\mathbf{2 8 . 4 8}$ \\
& NC (\%) & 38.80 & 61.90 & 57.71 & $\mathbf{6 5 . 3 1}$ & 62.87 & 28.77 \\
& B & $\mathbf{1 . 1 4}$ & 1.32 & 1.28 & 1.33 & 1.29 & 1.30 \\
& WEI (\%) & 66.33 & $\mathbf{7 4 . 5 7}$ & 72.36 & 74.3 & 73.90 & 73.84 \\
\hline \multirow{3}{*}{$3 \mathrm{~km}$} & POD (\%) & 89.90 & $\mathbf{9 3 . 0 7}$ & 90.96 & 88.95 & 90.61 & 90.88 \\
& FAR (\%) & 26.70 & 29.08 & 28.63 & $\mathbf{2 6 . 6 3}$ & 26.69 & 27.30 \\
& NC (\%) & $\mathbf{6 2 . 8 0}$ & 62.74 & 59.61 & 61.23 & 58.20 & 59.38 \\
& B & 1.23 & 1.31 & 1.27 & $\mathbf{1 . 2 1}$ & 1.24 & 1.25 \\
\hline
\end{tabular}

The smaller errors are given in bold.

TABLE 6: Statistics of the point quantitative verification for every ten, simulated with CPS and without CPS in domain 3.

\begin{tabular}{|c|c|c|c|c|c|c|c|c|c|c|}
\hline \multirow{2}{*}{ CPS use } & \multirow{2}{*}{ Statistics } & \multicolumn{9}{|c|}{ Tens } \\
\hline & & 1 & 2 & 3 & 4 & 5 & 6 & 7 & 8 & 9 \\
\hline \multirow{3}{*}{ CPS } & B (mm/day) & 6.04 & 4.55 & 1.76 & 2.64 & 5.636 & 5.97 & 3.69 & -1.06 & 3.97 \\
\hline & RMSE (mm/day) & 9.66 & 9.20 & 7.72 & 7.95 & 11.01 & 10.75 & 10.09 & 11.04 & 12.81 \\
\hline & MAE (mm/day) & 7.34 & 6.53 & 4.09 & 5.28 & 8.73 & 7.52 & 7.71 & 9.14 & 9.54 \\
\hline \multirow{3}{*}{ Not CPS } & B (mm/day) & 6.92 & 5.15 & 1.52 & 2.04 & 5.637 & 5.57 & 3.95 & -0.97 & 4.55 \\
\hline & RMSE (mm/day) & 11.09 & 9.93 & 6.71 & 7.28 & 11.30 & 10.42 & 9.80 & 10.58 & 13.26 \\
\hline & MAE (mm/day) & 8.46 & 7.22 & 3.94 & 4.95 & 8.97 & 7.19 & 7.73 & 8.58 & 9.95 \\
\hline
\end{tabular}

The smaller errors are given in bold.

3.2. Results of Punctual Verification: The Occurrence of Precipitation as a Categorical Binary Variable. Table 5 shows the statistics of categorical binary verification for the different configurations. In a general sense, it can be noticed that the levels of detection of the different configurations are greater than $85 \%$. However, the $B$ was greater than 1 , indicating that the model overestimates the presence of precipitations in the region. In the table, it is observed that, for the $6 \mathrm{~km}$ domain, the highest POD index (94.39\%) was obtained with the C_BMJ configuration, while the highest "negative correct" of $65.31 \%$ was obtained with the MP_MR configuration. The highest WEI was that of the C_BMJ scheme. The false alarms and $B$ indices show close values for all the configurations.

For the $3 \mathrm{~km}$ domain, also the C_BMJ configuration showed the best POD (93.07\%), with an NC of 62.74; however, the highest NC (62.8\%), and WEI (74.18) were shown by the control configuration. In general, for the two domains, the CTR, C_BMJ, and MP_MR configurations were the most effective.

Additional verifications were performed, only for the stations located in the northern, central, and southern 


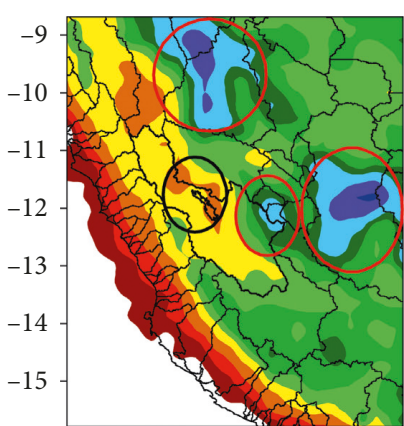

(a)

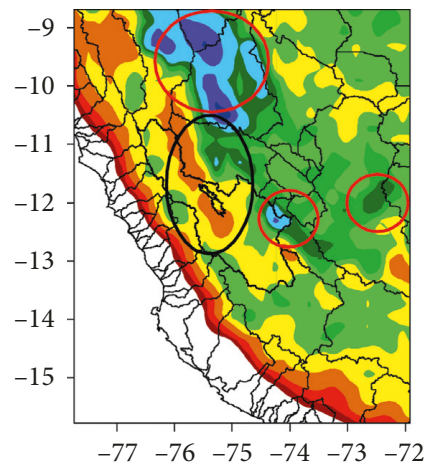

(b)

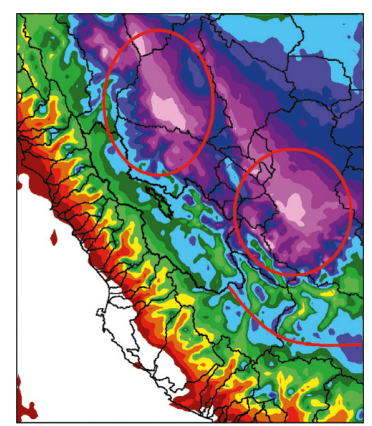

(c)

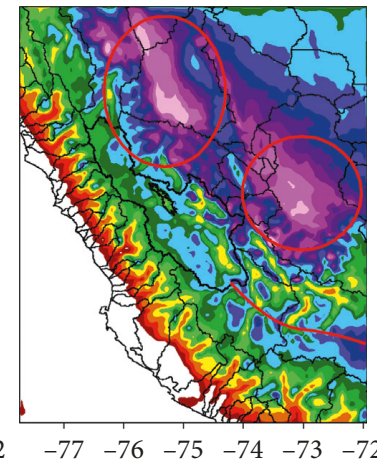

(d)

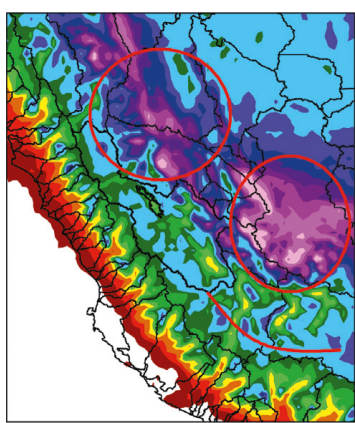

(e)

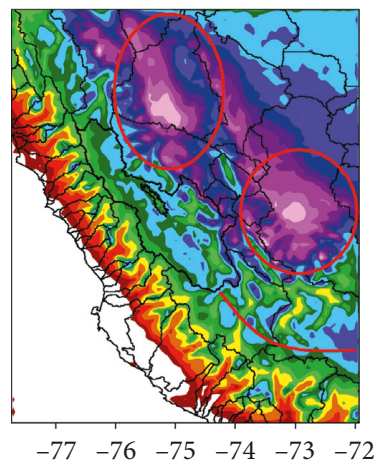

(f)

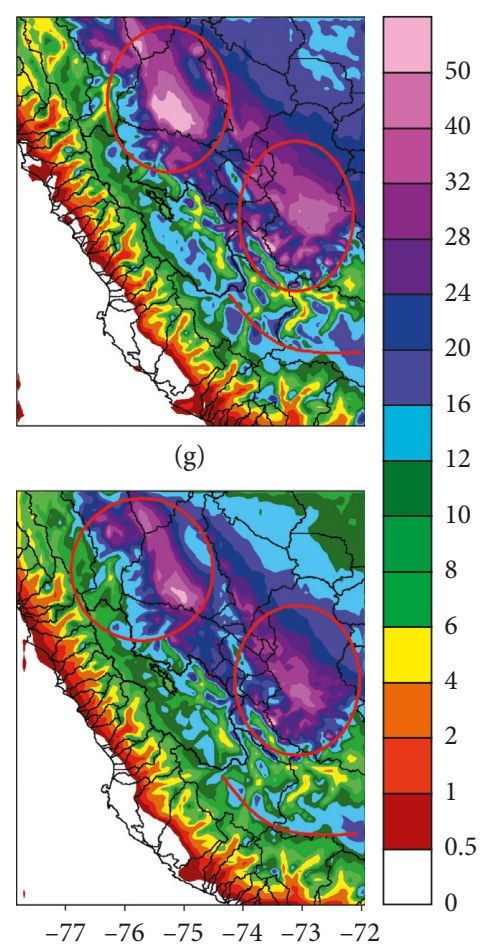

(h)

FIgURE 6: Spatial distribution of precipitation $(\mathrm{mm})$ for the $6 \mathrm{~km}$ domain, average of all periods studied: (a) TRMM, (b) PISCO, (c) CTR, (d) MP_LP, (e) C_BMJ, (f) BL_MYJ, (g) C_GRELL3, and (h) MP_MR.

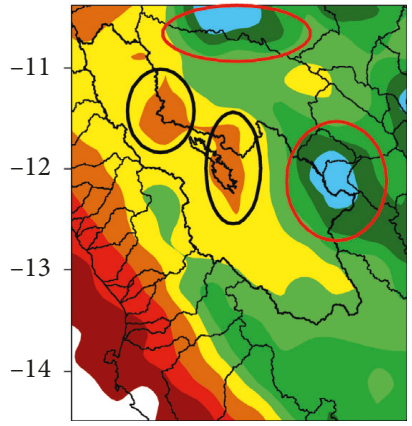

(a)

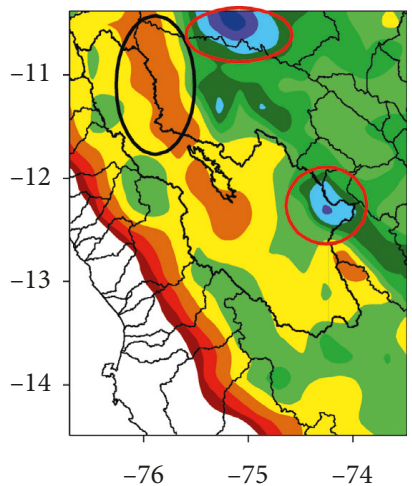

(b)

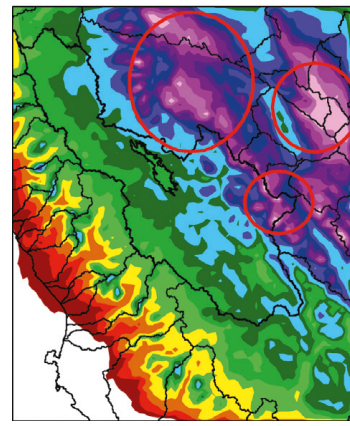

(c)

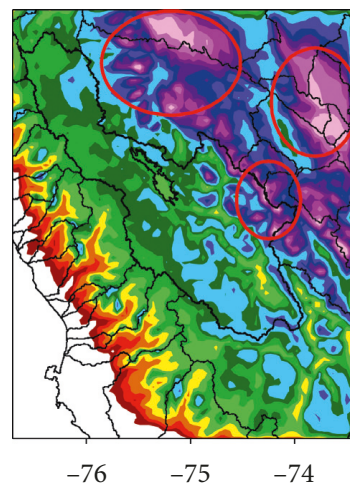

(d)

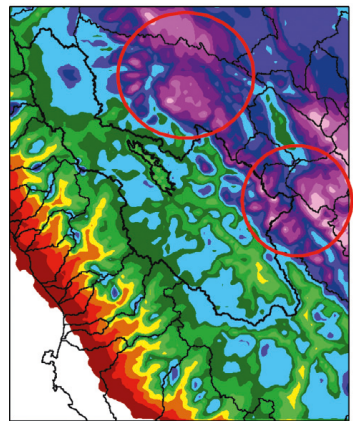

(e)

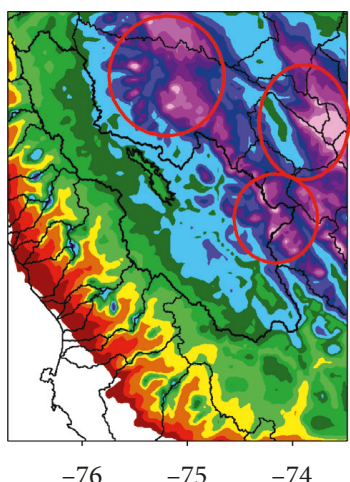

(f)

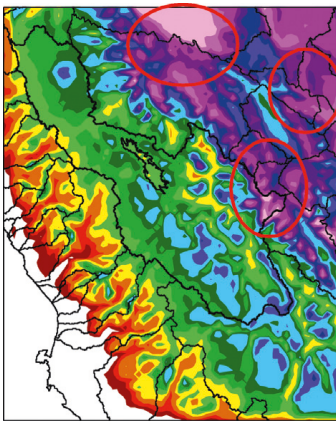

(g)

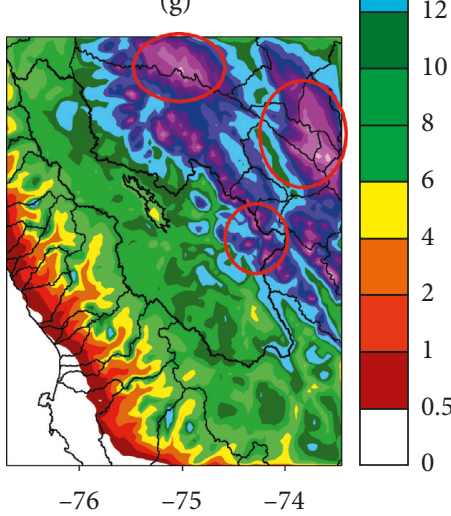

(h)

FIgURE 7: Spatial distribution of precipitation $(\mathrm{mm})$ for the $3 \mathrm{~km}$ domain, average of all periods studied: (a) TRMM, (b) PISCO, (c) CTR, (d) MP_LP, (e) C_BMJ, (f) BL_MYJ, (g) C_GRELL3, and (h) MP_MR. 


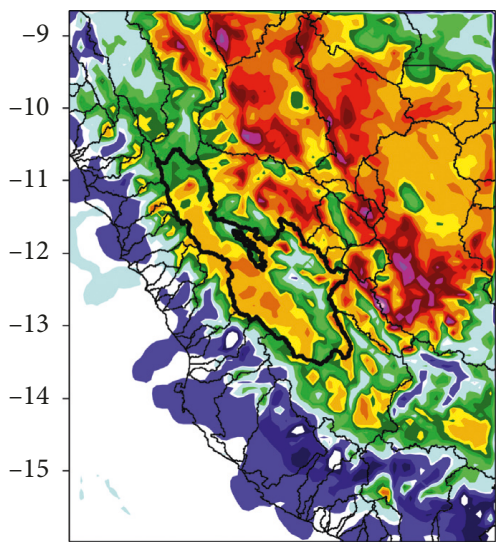

(a)

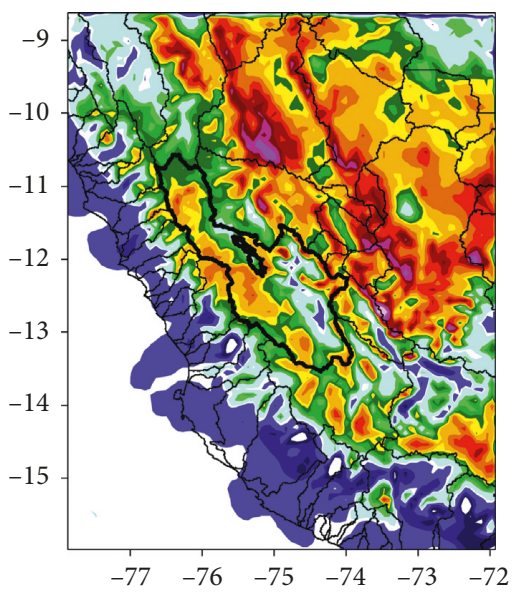

(b)

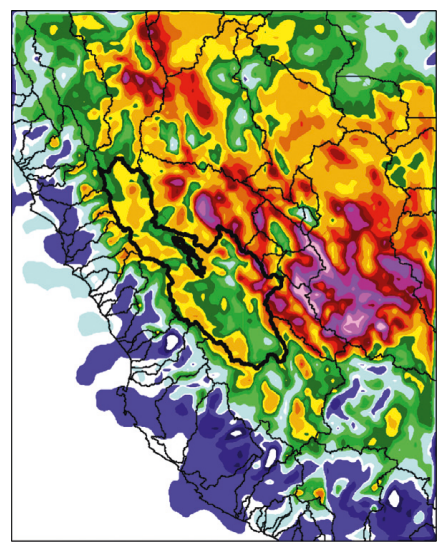

(c)

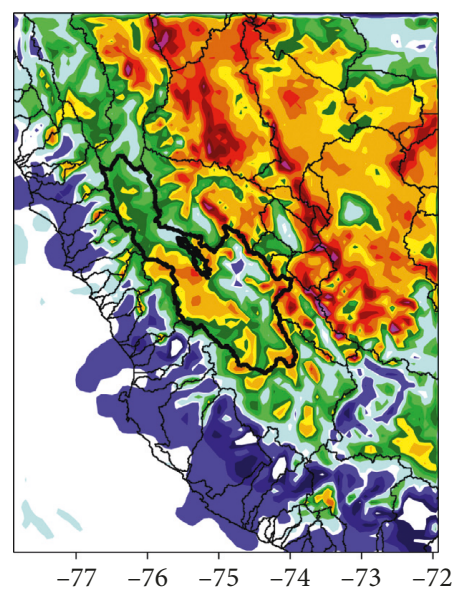

(d)

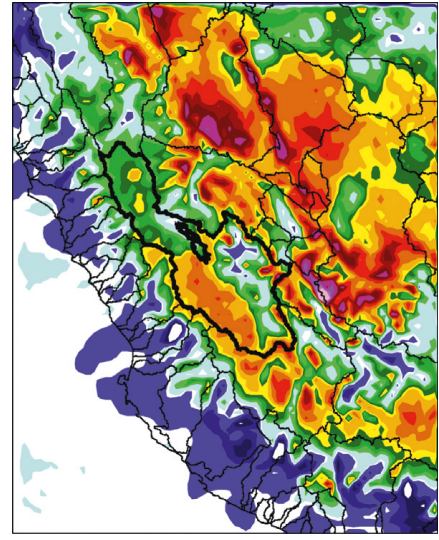

(e)

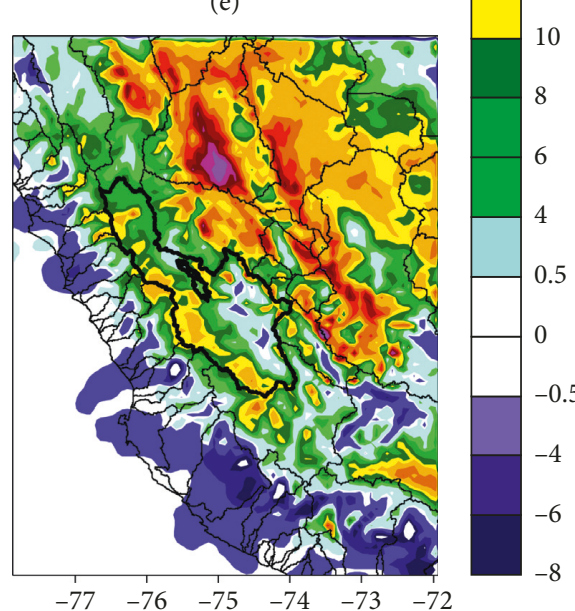

(f)

FIgURE 8: Spatial distribution of bias $B$ (mm/day) for the $6 \mathrm{~km}$ domain, corresponding to the second day of forecast: (a) CTR, (b) MP_LP, (c) C_BMJ, (d) BL_MYJ, (e) C_GRELL3, and (f) MP_MR.

regions of the basin, both quantitatively and qualitatively. However, the results obtained were similar to those shown considering all the basin stations, and we do not show them in this paper.

As we had mentioned in the introduction, from the results of the experiments with the different parameterizations, a new experiment was carried out using the MP_MR configuration (which showed the best $B$ and RMSE indicators for the entire forecast period) with the objective to verify the need or not to use CPS for the $3 \mathrm{~km}$ domain in the study region. In this sense, the results are shown in Table 6. In the table, the number of the tens corresponds to the order indicated in the Data and Methodology, and the best results for each ten are indicated in bold type. As can be seen, when looking at all the tens, the best results were obtained in some cases when CPS was used, and in others, when the cumulus scheme was deactivated. So, this result confirms the idea that it is not appropriate to assume that, for domains of less than $5 \mathrm{~km}$, the parameterization of clusters must be deactivated, as was concluded in [21].

\subsection{Results of the Verification of the Spatial Precipitation Field} Forecast. Figure 6 shows the average spatial distribution of the precipitation from the TRMM and PISCO databases and the model output for the different configurations used for the studied periods, corresponding to the $6 \mathrm{~km}$ domain. Regarding the data of TRMM and PISCO (Figures 6(a) and 6(b)), it can be seen that both databases show very similar patterns of precipitation. In this sense, the greater difference is that TRMM shows a maximum of rainfall around $12^{\circ} \mathrm{S}$ and $73^{\circ} \mathrm{W}$, which is less notorious in PISCO, due to the fact that in that region there are scarce meteorological stations. Two maximum precipitation regions have been highlighted in red circles in Figures 6 and 7 in the zone of Amazonia, to the east of the mountain range. Another precipitation maximum of smaller area (also surrounded by a red circle in the figures) is located at the southeast of the Mantaro Basin, which contour line is represented by a thick black line in the figure.

An elongated rainy area which starts from the south of the basin and extends towards the southeast has been indicated with a thick red curve in each figure. Another significant feature is the relative spatial rainfall minimum shown by PISCO along part of the eastern boundary of the basin, mostly in its northern half, indicated by black circles in Figures 6(a) and 6(b). In TRMM, this zone is less extended to the north.

The maps in Figures 6(c)-6(h) show the output of WRF for the different configurations used in the experiments. 


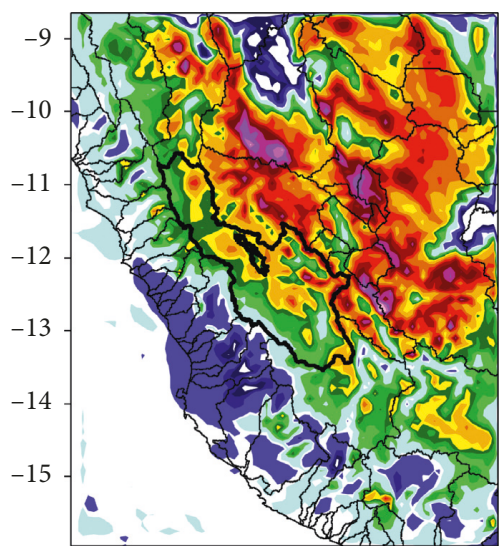

(a)

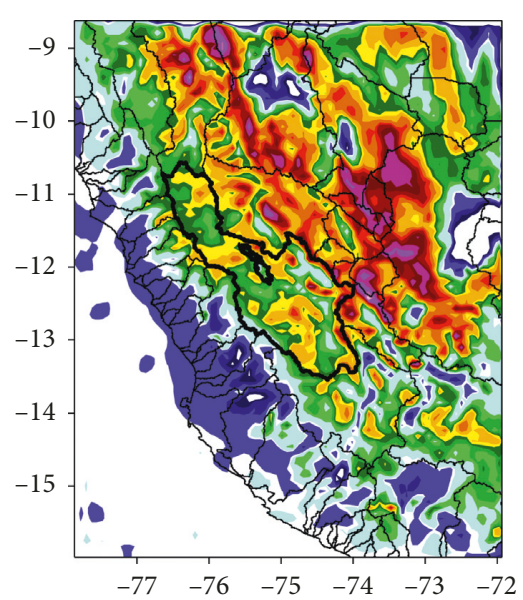

(b)

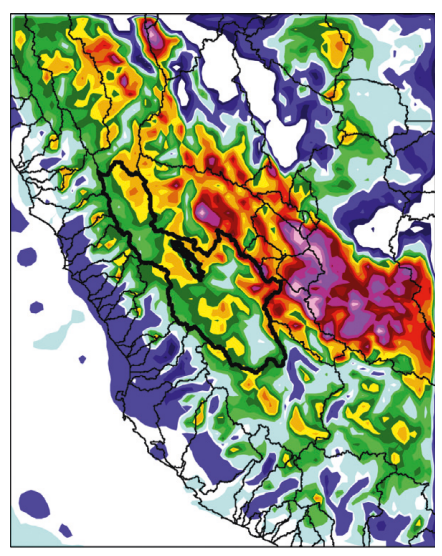

(c)

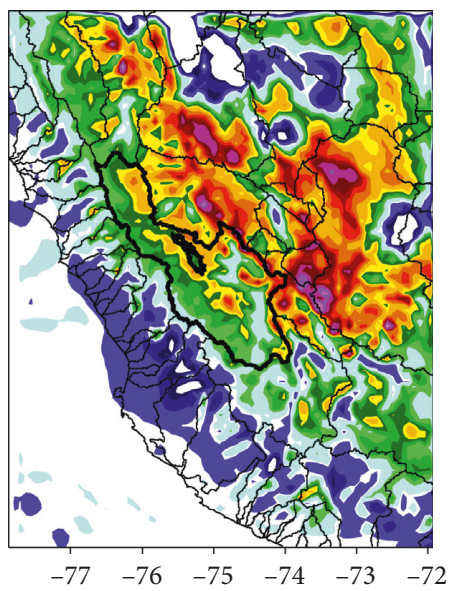

(d)

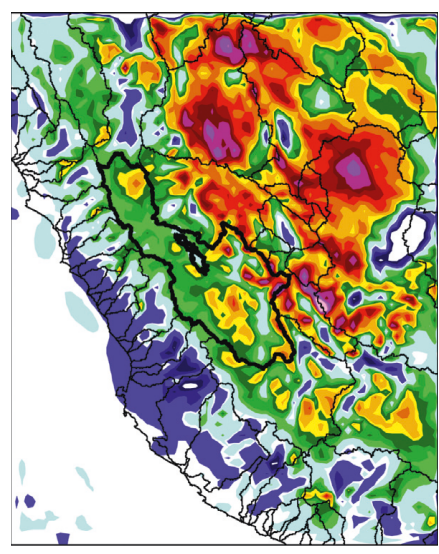

(e)

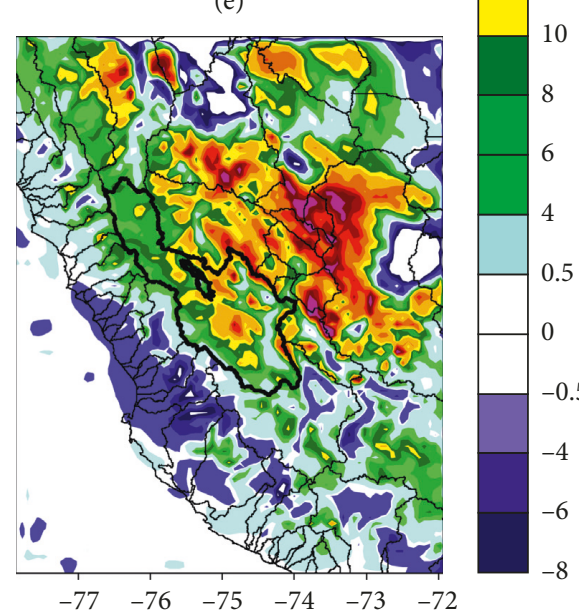

(f)

Figure 9: Spatial distribution of the $B$ ( $\mathrm{mm} /$ day) for the $6 \mathrm{~km}$ domain, corresponding to the sixth day of forecast: (a) CTR, (b) MP_LP, (c) C_BMJ, (d) BL_MYJ, (e) C_GRELL3, and (f) MP_MR.

It can be noticed that, in general, all configurations reproduced correctly the precipitation patterns shown by PISCO and TRMM but overestimating the reference accumulations, shown in Figures 6(a) and 6(b), mainly on the eastern slope of the mountain range (hot spots). However, it must be mentioned that it has been found [43] that in this sector of the Peruvian mountain range, TRMM underestimates precipitation by at least $40 \%$, so that the overestimation of WRF relative to the "real precipitation value" in that case could be much lower. The configuration which best reproduced the maximum in $12^{\circ} \mathrm{S}-72^{\circ} \mathrm{W}$, relative to TRMM was C_BMJ.

It must be pointed out that none of the configurations reproduced the less rainy strip shown by PISCO and, to a lesser extent, also by TRMM, along the eastern limit of the basin in its northern half (black circles in Figures 6(a) and 6(b)). In fact, WRF produces more precipitation in the eastern sector of the basin than in the west, which matches the observations only for the southern half of the basin.

Figure 7 shows the zoomed-in view of TRMM and PISCO precipitation patterns and WRF output for the $3 \mathrm{~km}$ domain. In TRMM and PISCO (Figures 7(a) and 7(b)), local maxima and minimum of precipitation are indicated as in Figure 6, using red circles fox maxima and with a black circle for the minimum of precipitation in the eastern limit of the northern half of the basin, practically covering the valley (which is represented by a small closed black contour near the western boundary of the basin). In a general sense, it is observed that the basin in its southern half is rainier towards the east, while in the northern half it is rainier towards the west, which can be seen more clearly in the PISCO data. It can also be noticed, more clearly in TRMM, that the south of the valley is drier than the north.

As shown in Figures 7(c)-7(h), the model reproduces the precipitation nuclei to the east of the basin, although in a general sense it overestimates accumulated rainfall. It can be seen that toward the southern half of the basin, the MP_MR configuration reproduced more clearly the fact that the eastern sector is rainier than the western. The CTR configuration also reproduces this pattern but much more extended to the west. The rest of the configurations were less precise in this regard. In general, the MP_MR configuration was the one reproducing best the spatial distribution of rainfall in the south of the basin. For the northern half of the basin, all the configurations reflected the eastern sector as rainier than the western, which does not reproduce the patterns in TRMM and PISCO.

From the above considerations, it can be concluded that for the $6 \mathrm{~km}$ domain, the precipitation pattern shown by 


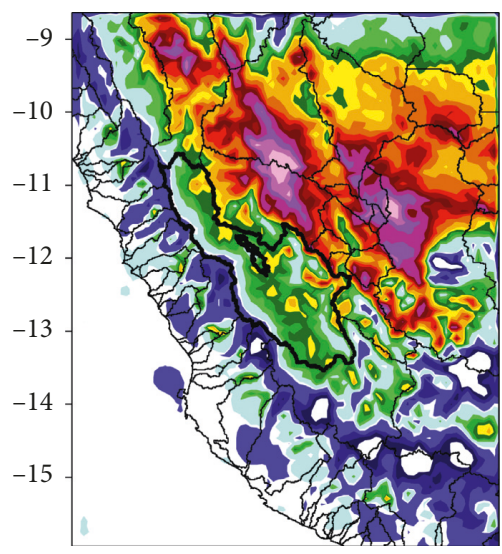

(a)

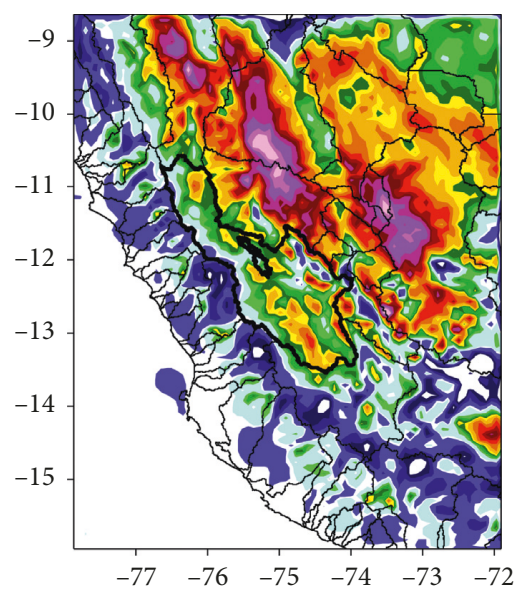

(b)

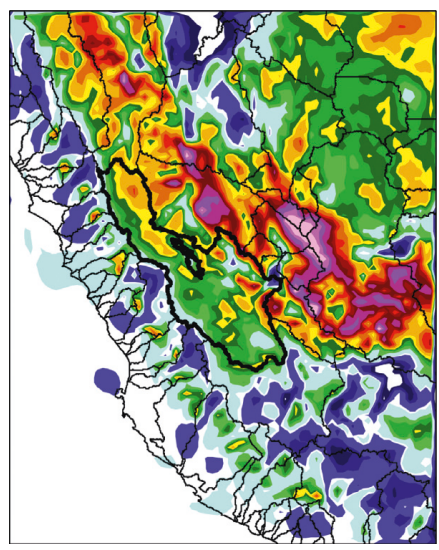

(c)

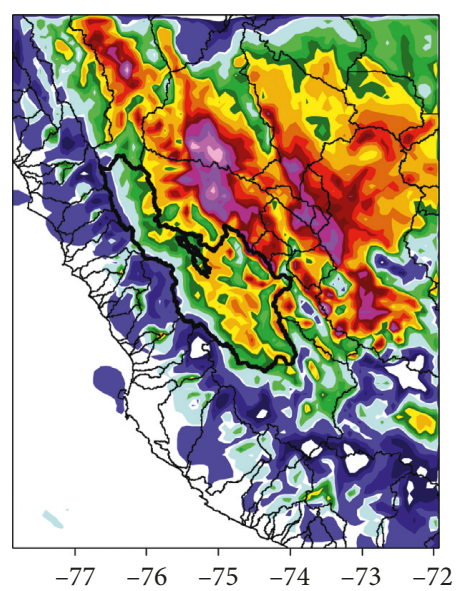

(d)

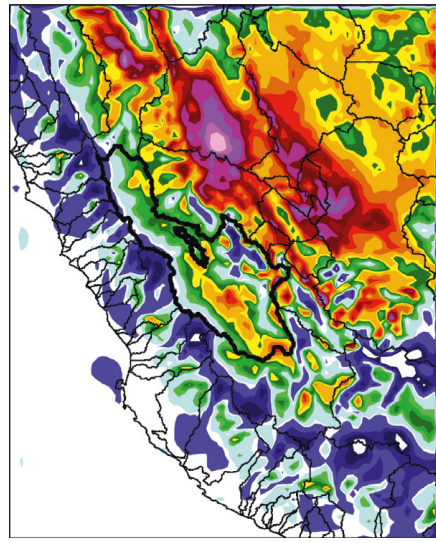

(e)

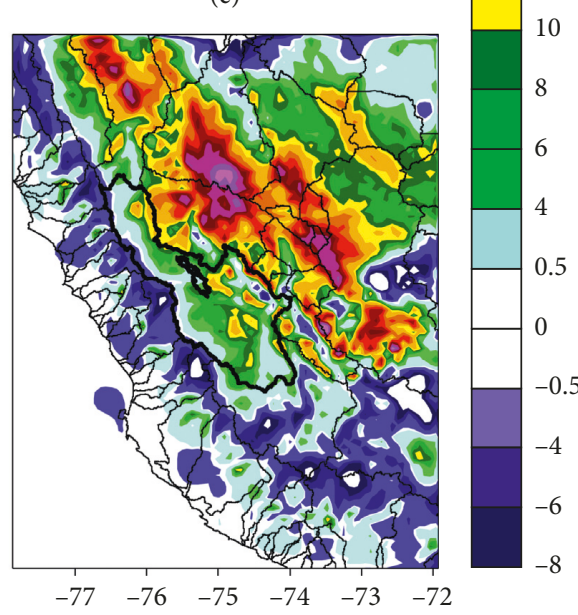

(f)

Figure 10: Spatial distribution of the $B$ ( $\mathrm{mm}$ /day) for the $6 \mathrm{~km}$ domain, corresponding to the tenth day of forecast: (a) CTR, (b) MP_LP, (c) C_BMJ, (d) BL_MYJ, (e) C_GRELL3, and (f) MP_MR.

C_BMJ matches better the PISCO and TRMM patterns, although the MP_MR configuration seems to overestimate less than the rest. For domain 3, all the configurations reflected the maximum precipitation to the east of the basin, although the MP_MR configuration better reflected the spatial distribution of rainfall in the southern half of the basin. Towards the northern half of the basin, none of the configurations reproduces well the observed patterns of TRMM and PISCO.

In order to quantify the spatial distribution of the bias of the model, the bias $(B)$ maps of both domains were made for different forecast horizons. However, in this case, bias will be calculated relative to TRMM data, considering that PISCO reflects a significant deficit of rainfall to the southeast of the basin. Figure 8 shows the spatial $B$ distribution for the second day of forecast of the $6 \mathrm{~km}$ domain, which confirms that in general all the configurations overestimate the precipitation; however, it is noticed that it underestimates both for the western sector of the mountain range and for the coast, although this sector of the Andes is much less rainy than its eastern sector. In this case, it can be seen that the MP_MR configuration produces the least overestimation, as we had observed in the general picture.
Figures 9 and 10 show the results for the sixth and tenth days of forecast, where it is visually noticed that the MP_MR configuration has the lowest bias. In this case, it is significant that, for the sixth day the configuration, C_BMJ shows a noticeable improvement in relation to the second, specifically in the Amazonia zone, with insignificant overestimations.

Figures 11-13 are analogous to Figures 8-10, but for the $3 \mathrm{~km}$ domain. As for the $6 \mathrm{~km}$ domain, in this case, the model overestimates precipitation, and even in the interior of the basin, some of the configurations overestimate more than in the $6 \mathrm{~km}$ domain.

For this domain, in all cases, it is also noticed that MP_MR showed the best results, but with the particularity that outside the basin, $B$ increased with forecast horizon, while the behavior inside the basin was more stable.

Thus, from a spatial point of view, the model clearly shows an overestimation in the Mantaro basin and, in general, in the entire eastern sector of the ridge, while in its western sector, underestimation of precipitation was observed. From the spatial point of view, it is confirmed that MP_MR was the configuration with the lowest overestimation, behaving in a stable way from the second to the tenth forecast day, with a moderate increase in RMSE as the forecast horizon increased. 


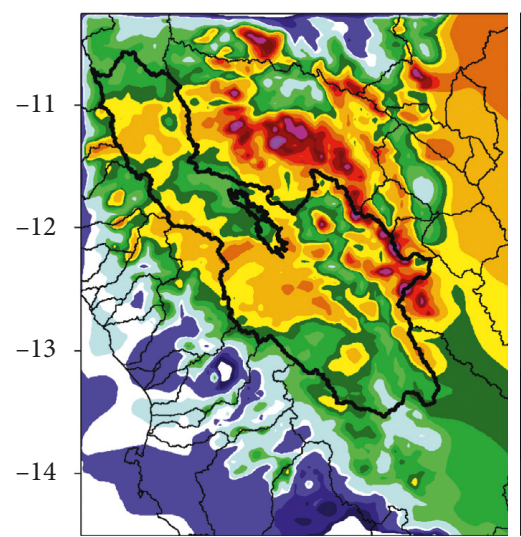

(a)

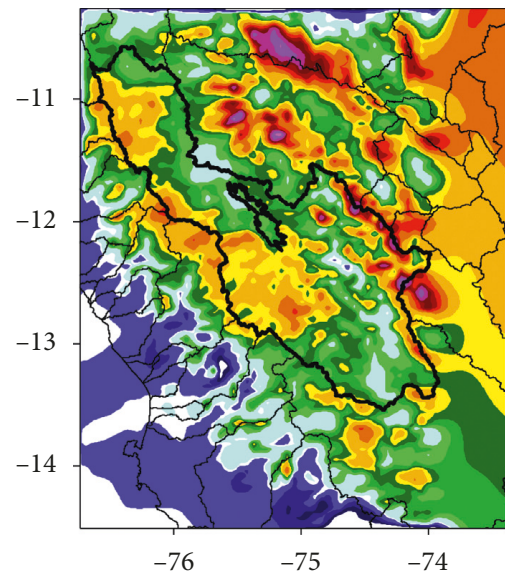

(b)

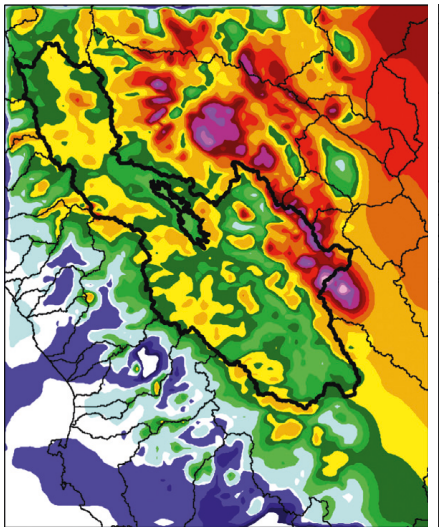

(c)

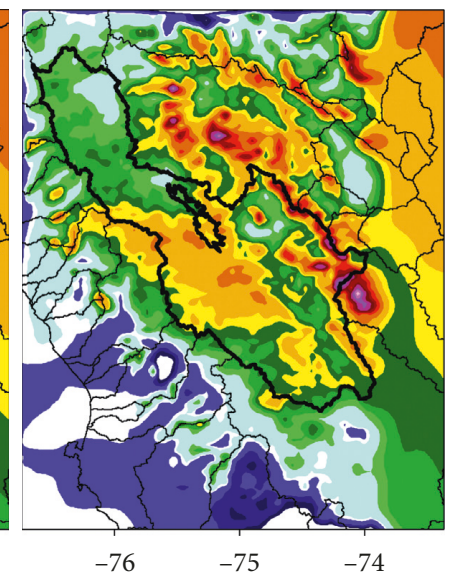

(d)
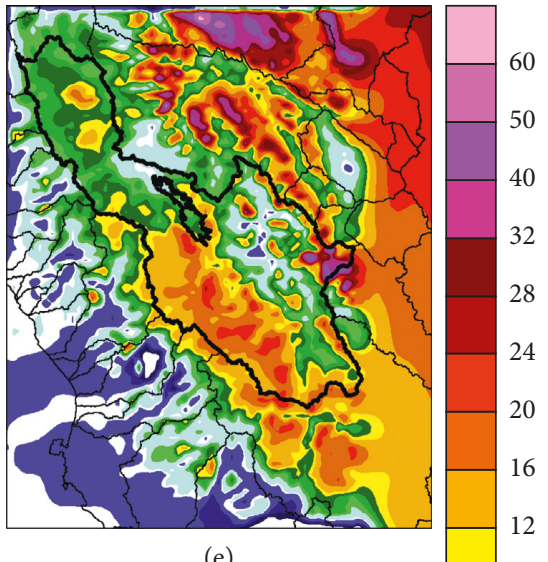

(e)

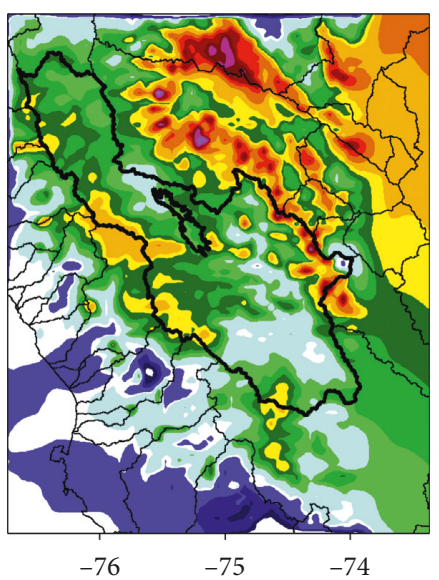

(f)

FIgURE 11: Spatial distribution of $B$ ( $\mathrm{mm} /$ day) for the $3 \mathrm{~km}$ domain, corresponding to the second day of forecast: (a) CTR, (b) MP_LP, (c) C_BMJ, (d) BL_MYJ, (e) C_GRELL3, and (f) MP_MR.

From the above arguments, it can be concluded that the sensitivity to the microphysical scheme was higher than to the convective scheme, which indicates the importance of the microphysics parameterization in WRF for precipitation forecasting tasks in the study region. As an example, Figure 14 shows the vertical profile of rain water mixing ratio (Qrain) produced by the 3 microphysical schemes used for the Huayao station and, in parentheses, the predicted rainfall in each case, averaged for all tens used in study. It can be noticed the correspondence between "Qrain" and predicted precipitation.

Similar to the results obtained in this research, in [25], a sensitivity study was made of simulated monsoon precipitation to the cloud microphysics schemes in WRF for the summer period in the valley of Langtang, Himalaya. It was obtained that, in a general sense, the model underestimated the accumulated precipitation in 10 days, and also the Morrison microphysical scheme showed the best results.

Our results for the Andes are consistent with the finding of [26], where it was concluded that, in general, the doublemoment Morrison's scheme represents more correctly the microphysics processes in the Himalaya, possibly because of the better representation of the warm and cold processes related with the formation and evolution of the parameters of particle size distributions, in comparison with onemoment parameterizations.

\section{Summary and Conclusions}

Simulations were developed with the regional model WRF, for domains of 18,6 , and $3 \mathrm{~km}$ of spatial resolution, with the main objective of determining the ability of several configurations of the model to forecast the field of precipitation in the short and medium terms in the complex orography conditions of the Central Andes of Peru. The initial and boundary conditions were generated by the NCEP "Global Operational Analysis," final analysis FNL, every 6 hours, with horizontal resolution of $1^{\circ} \times 1^{\circ}$. The behavior of the numerical forecasts for the first 10 days of simulation was analyzed, concluding that the mean square error for the higher resolution domains increases with the simulation term, in general until the tenth day. The lowest mean square error was obtained for the MP_MR configuration (Morrison for microphysics and Grell-Freitas for convection) during the whole period, remaining with little variation after the sixth forecast day. This configuration also provided the lowest positive bias, although in all cases a clear overestimation of rainfall was observed. 


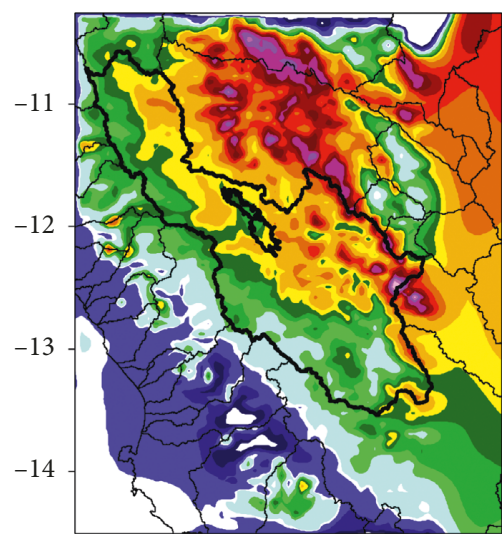

(a)

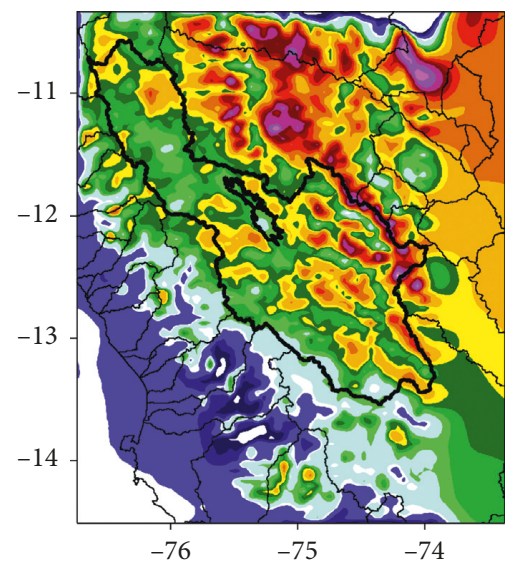

(b)

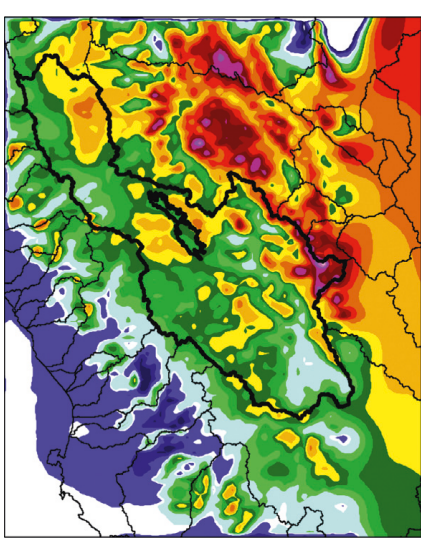

(c)

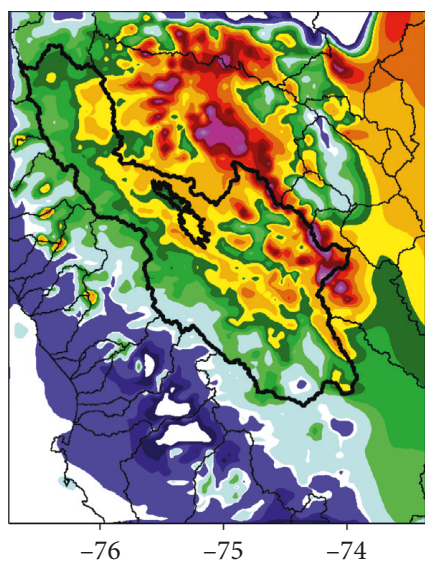

(d)

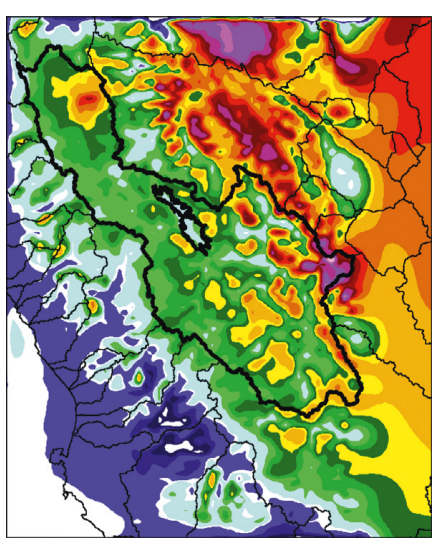

(e)

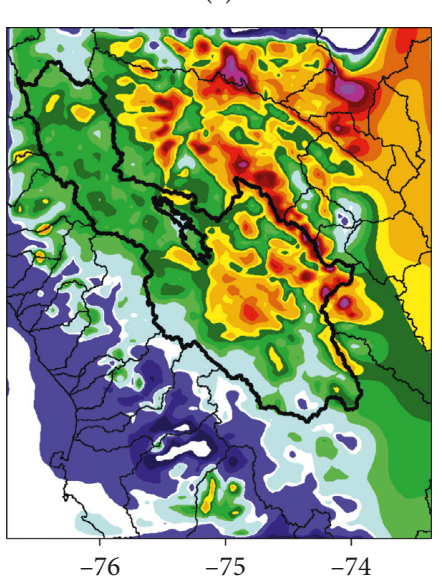

(f)

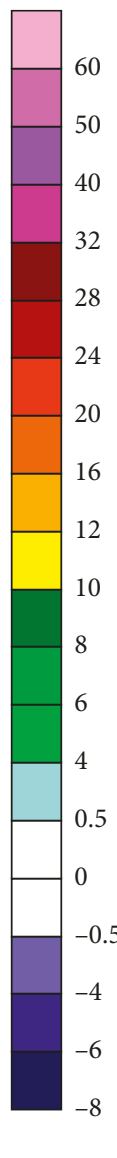

FIGURE 12: Spatial distribution of the $B$ ( $\mathrm{mm} /$ day) for the $3 \mathrm{~km}$ domain, corresponding to the sixth day of forecast: (a) CTR, (b) MP_LP, (c) C_BMJ, (d) BL_MYJ, (e) C_GRELL3, and (f) MP_MR.

The point verification showed that, in general, all the tested configurations overestimate rainfall in the region. In this sense, the MP_MR configuration performed better, according to the bias and RMSE indicators, which alludes not only to the entire forecast period but also to all the forecast terms. It is important to notice that from the sixth day on, the RMSE for MP_MR did not increase more, which does not apply to the rest of the schemes used. Another important result in this case is that the C_BMJ configuration (Thompson microphysics and Betts-Miller-Janjic convection) showed poor results for the $18 \mathrm{~km}$ domain, so it is recommended not to use it in this region for low-resolution domains.

The categorical binary verification showed detection rates of precipitation above $85 \%$ in all cases. The $B$ was all positive, which indicates that the model overestimated the presence of rainfall in the region. In general, the configurations showing the best indicators were $\mathrm{C}_{-} \mathrm{BMJ}$ and MP_MR.

The spatial verification showed that all the schemes produced rainfall distribution patterns quite similar to those of TRMM and PISCO, although in all cases with positive bias in precipitation accumulations. In this sense, the configuration that less overestimated precipitation was MP_MR. It also reproduced better precipitation pattern in the southern half of the basin. Regarding the northern half of the basin, all the configurations showed the eastern sector as rainier than the west, which does not agree with TRMM and PISCO.

The bias spatial distribution generally showed positive values in the basin and in general throughout the eastern sector of the Andes and the Amazonia, while towards the west of the Andes and the coast, it showed values close to zero or negative. The configuration with lower bias was MP_MR, which also showed the lowest values of RMSE, in both cases for the two domains analyzed. The C_BMJ configuration, although with higher bias and RMSE, showed improvement for the greater terms, which is an indicator to be taken into account for its use in longer-term forecast.

Based on the results, it is concluded that the MP_MR and C_BMJ configurations were the ones with the best results in a general sense. Consequently, the MP_MR configuration is recommended for short- and medium-term rainfall forecasting tasks in the Central Andes of Peru and particularly in the Mantaro basin. The Betts-Miller-Janjic cumulus parameterization will be further investigated by the authors for the region in combination with other physical schemes to explore its possible application potential in medium range precipitation forecasting, using multiconfiguration ensembles. It was also concluded that the activation or not of cumulus parametrization for the domain of $3 \mathrm{~km}$ resolution is not relevant for the precipitation forecast in the study region. 


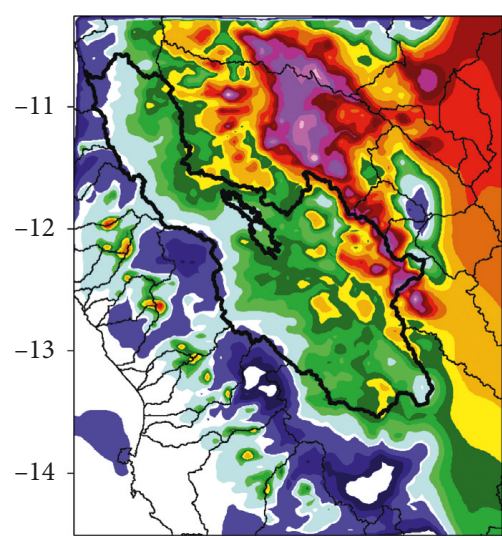

(a)

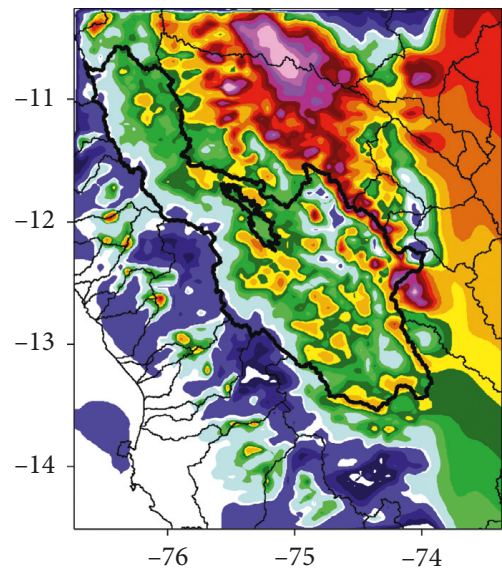

(b)

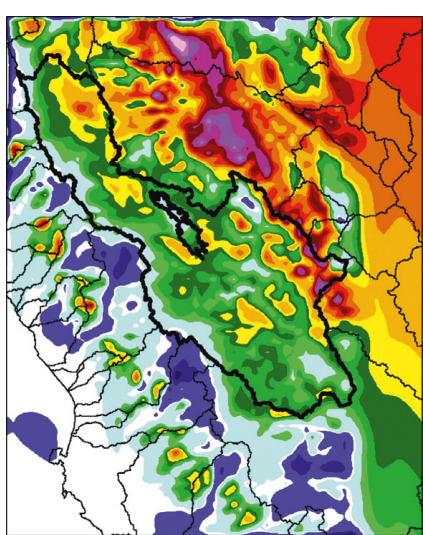

(c)

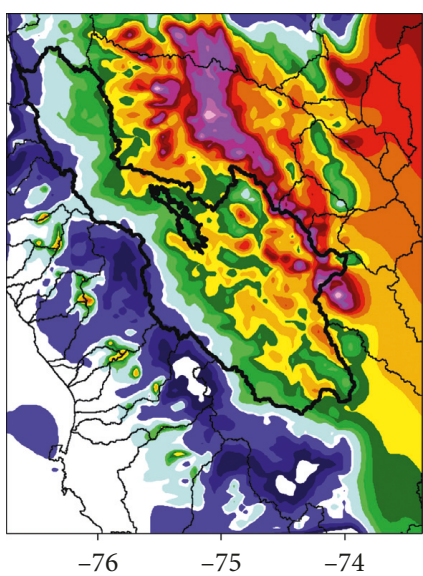

(d)

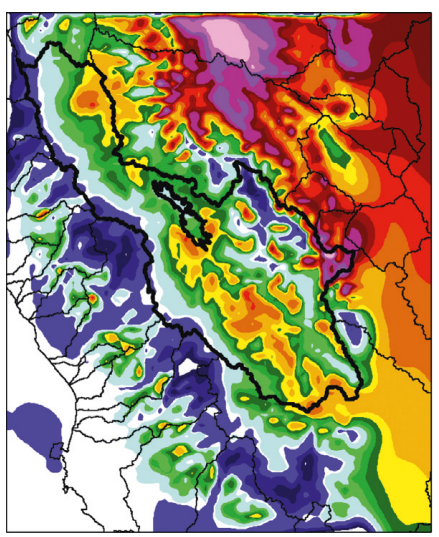

(e)

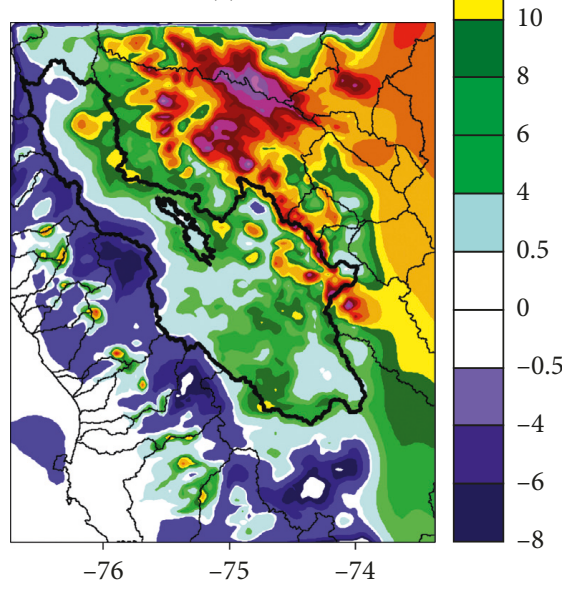

(f)

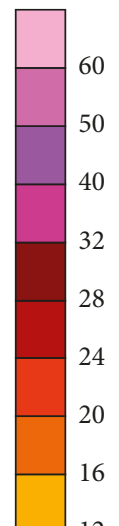

13. Spatial distribution of the $B(\mathrm{~mm} /$ day) for the $3 \mathrm{~km}$ domain, corresponding to the tenth day of forecast: (a) CTR, (b) MP LP (c) C_BMJ, (d) BL_MYJ, (e) C_GRELL3, and (f) MP_MR.

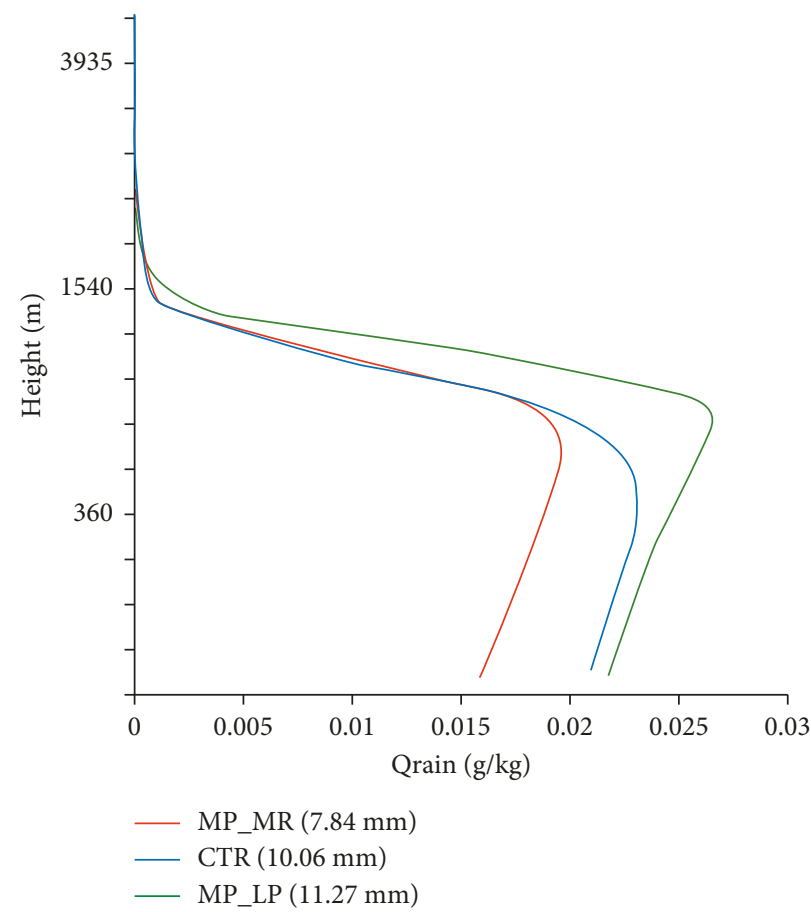

FIGURE 14: Vertical profile of "rain water mixing ratio (Qrain)" produced by the 3 microphysical schemes used for the Huayao station and, in parentheses, the predicted rainfall in each case, average of 10 study cases taken randomly. 


\section{Data Availability}

The data used to support the findings of this study are available from the corresponding author upon request.

\section{Conflicts of Interest}

The authors declare that there are no conflicts of interest regarding the publication of this paper.

\section{Acknowledgments}

The present study comes under the project "MAGNET-IGP: Strengthening the research line in physics and microphysics of the atmosphere (Agreement no. 010-2017-FONDECYT)." The authors would like to thank the CONCYTEC, Peru, for financial support and Inter-American Institute for Cooperation on Agriculture (IICA) for administrative support. This work was done using computational resources, HPC-Linux Cluster, from Laboratorio de Dinámica de Fluidos Geofísicos Computacionales at Instituto Geofísico del Perú (Grants 101-2014-FONDECYT, SPIRALES2012 IRD-IGP, Manglares IGP-IDRC, and PP068 program). The authors also thank NASA for TRMM precipitation data, NCEP for FNL analysis data, and SENAMHI for observational precipitation data.

\section{References}

[1] Y. H. Kuo, J Bresch, M. D. Cheng et al., "Summary of a mini workshop on cumulus parameterization for mesoscale models," Bulletin of the American Meteorological Society, vol. 78, no. 3, pp. 475-491, 1997.

[2] W. Skamarock, J. Klemp, J. Dudhia et al., A Description of the Advanced Research WRF Version 3, NCAR Technical Note, NCAR/TN-468+STR, National Center for Atmospheric Research (NCAR), Mesoscale and Microscale Meteorology Division, Boulder, CO, USA, 2008.

[3] Y. Silva, K. Takahashi, and R. Chávez, "Dry and wet rainy seasons in the Mantaro river basin (Central Peruvian Andes)," Advances in Geosciences, vol. 14, pp. 261-264, 2008.

[4] P. Aceituno, "On the functioning of the southern oscillation in the South American, sector. Part II. Upper-air circulation," Journal of Climate, vol. 2, no. 4, pp. 341-355, 1989.

[5] M. Vuille, G. Kaser, and I. Juen, "Glacier mass balance variability in the Cordillera Blanca, Peru and its relationship with climate and the large-scale circulation," Global and Planetary Change, vol. 62, no. 1-2, pp. 14-28, 2008.

[6] R. Garreaud, "The Andes climate and weather," Advances in Geosciences, vol. 22, pp. 3-11, 2009.

[7] A. G. Martínez, E. Núñez, Y. Silva et al., "Vulnerability and adaptation to climate change in the Peruvian Central Andes: results of a pilot study," in Proceedings of the International Conference on Southern Hemisphere Meteorology and Oceanography (ICSHMO), pp. 297-305, Foz do Iguaçu, PR, Brazil, April 2006.

[8] B. S. Barrett, R. Garreaud, and M. Falvey, "Effect of the Andes cordillera on precipitation from a midlatitude cold front," Monthly Weather Review, vol. 137, no. 9, pp. 3092-3109, 2009.

[9] M. Viale and F. A. Norte, "Strong cross-barrier flow under stable conditions producing intense winter orographic precipitation: a case study over the subtropical central Andes," Weather and Forecasting, vol. 24, no. 4, pp. 1009-1031, 2009.

[10] P. A. Jiménez, J. Dudhia, J. F. González-Rouco et al., "An evaluation of WRF's ability to reproduce the surface wind over complex terrain based on typical circulation patterns," Journal of Geophysical Research: Atmospheres, vol. 118, no. 14, pp. 7651-7669, 2013.

[11] T. M. Weckwerth, I. J. Bennett, L. Jay Miller et al., “An observational and modeling study of the processes leading to deep, moist convection in complex terrain," Monthly Weather Review, vol. 142, no. 8, pp. 2687-2708, 2014.

[12] C. Junquas, K. Takahashi, T. Condom et al., "Understanding the influence of orography on the precipitation diurnal cycle and the associated atmospheric processes in the central Andes," Climate Dynamics, vol. 50, no. 11-12, pp. 3995-4017, 2017.

[13] Z. I. Janjic, "Nonsingular implementation of the MellorYamada level 2.5 scheme in the NCEP meso model," NCEP Office Note, no. 437, p. 61, 2002.

[14] S.-Y. Hong, Y. Noh, and J. Dudhia, "A new vertical diffusion package with an explicit treatment of entrainment processes," Monthly Weather Review, vol. 134, no. 9, pp. 2318-2341, 2006.

[15] Z. I. Janjic, “The step-mountain eta coordinate model: further developments of the convection, viscous sublayer, and turbulence closure scheme," Monthly Weather Review, vol. 122, no. 5, pp. 927-945, 1994.

[16] G. A. Grell and S. R. Freitas, "A scale and aerosol aware stochastic convective parameterization for weather and air quality modeling," Atmospheric Chemistry and Physics, vol. 14, no. 10, pp. 5233-5250, 2014.

[17] G. Thompson, P. R. Field, R. F. Rasmussen, and W. D. Hall, "Explicit forecasts of winter precipitation using an improved bulk microphysics scheme. Part II: implementation of a new snow parameterization," Monthly Weather Review, vol. 136, no. 12, pp. 5095-5115, 2008.

[18] H. Morrison, G. Thompson, and V. Tatarskii, "Impact of cloud microphysics on the development of trailing stratiform precipitation in a simulated squall line: comparison of oneand two-moment schemes," Monthly Weather Review, vol. 137, no. 3, pp. 991-1007, 2009.

[19] Y. L. Lin, R. D. Farley, and H. D. Orville, "Bulk parametrization of the snow field in a cloud model," Journal of Climate and Applied Meteorology, vol. 22, no. 6, pp. 1065-1092, 1983.

[20] J. Done, C. A. Davis, and M. Weisman, "The next generation of NWP: explicit forecasts of convection using the weather research and forecasting (WRF) model," Atmospheric Science Letters, vol. 5, no. 6, pp. 110-117, 2004.

[21] E. K. Gilliland and C. M. Rowe, "A comparison of cumulus parameterization schemes in the WRF model," in Proceedings of the 87th AMS Annual Meeting and 21st Conference on Hydrology, San Antonia, TX, USA, January 2007.

[22] T. G. Farr, P. A. Rosen, E. Caro et al., "The shuttle radar topography mission," Reviews of Geophysics, vol. 45, no. 2, 2007.

[23] E. Rodriguez, C. S. Morris, and J. E. Belz, "A global assessment of the SRTM performance," Photogrammetric Engineering and Remote Sensing, vol. 72, no. 3, pp. 249-260, 2006.

[24] D. B. Gesch, K. L. Verdin, and S. K. Greenlee, "New land surface digital elevation model covers the earth," Eos, Transactions American Geophysical Union, vol. 80, no. 6, pp. 69-70, 1999.

[25] A. Orr, C. Listowski, M. Cottet et al., "Sensitivity of simulated summer monsoonal precipitation in Langtang Valley, Himalaya, to cloud microphysics schemes in WRF," Journal of 
Geophysical Research: Atmospheres, vol. 122, no. 12, pp. 6298-6318, 2017.

[26] R. K. Shrestha, P. J. Connolly, and M. W. Gallagher, "Sensitivity of WRF cloud microphysics to simulations of a convective storm over the Nepal Himalayas," The Open Atmospheric Science Journal, vol. 11, no. 1, pp. 29-43, 2017.

[27] M. Rajeevan, A. Kesarkar, S. B. Thampi, T. N. Rao, B. Radhakrishna, and M. Rajasekhar, "Sensitivity of WRF cloud microphysics to simulations of a severe thunderstorm event over Southeast India," Annales Geophysicae, vol. 28, no. 2, pp. 603-619, 2010.

[28] Y. G. Mayor and M. D. S. Mesquita, "Numerical simulations of the 1 May 2012 deep convection event over Cuba: sensitivity to cumulus and microphysical schemes in a highresolution model," Advances in Meteorology, vol. 2015, Article ID 973151, 16 pages, 2015.

[29] M Tewari, F. Chen, W. Wang et al., "Implementation and verification of the unified NOAH land surface model in the WRF model," in Proceedings of the 20th Conference on Weather Analysis and Forecasting/16th Conference on Numerical Weather Prediction, pp. 11-15, Seattle, WA, USA, January 2004.

[30] C. A. Paulson, "The mathematical representation of wind speed and temperature profiles in the unstable atmospheric surface layer," Journal of Applied Meteorology, vol. 9, no. 6, pp. 857-861, 1970.

[31] A. J. Dyer and B. B. Hicks, "Flux-gradient relationships in the constant flux layer," Quarterly Journal of the Royal Meteorological Society, vol. 96, no. 410, pp. 715-721, 1970.

[32] E. K. Webb, "Profile relationships: the log-linear range, and extension to strong stability," Quarterly Journal of the Royal Meteorological Society, vol. 96, no. 407, pp. 67-90, 1970.

[33] D. Zhang and R. A. Anthes, "A high-resolution model of the planetary boundary layer-sensitivity tests and comparisons with SESAME-79 data," Journal of Applied Meteorology, vol. 21, no. 11, pp. 1594-1609, 1982.

[34] A. C. M. Beljaars, "The parameterization of surface fluxes in large-scale models under free convection," Quarterly Journal of the Royal Meteorological Society, vol. 121, no. 522, pp. 255-270, 1994.

[35] M. J. Iacono, J. S. Delamere, E. J. Mlawer et al., "Radiative forcing by long-lived greenhouse gases: calculations with the AER radiative transfer models," Journal of Geophysical Research, vol. 113, p. D13, 2008.

[36] E. J. Mlawer, S. J. Taubman, P. D. Brown, M. J. Iacono, and S. A. Clough, "Radiative transfer for inhomogeneous atmospheres: RRTM, a validated correlated- $\mathrm{k}$ model for the longwave," Journal of Geophysical Research: Atmospheres, vol. 102, no. 14, pp. 16663-16682, 1997.

[37] R. M. Goody and Y. L. Yung, Atmospheric Radiation: Theoretical Basis, Oxford University Press, Oxford, UK, 1995, https://www.bookdepository.com/Atmospheric-RadiationTheoretical-Basis-R-M-Goody/9780195102918.

[38] G. P. Cressman, "An operational objective analysis system," Monthly Weather Review, vol. 87, no. 10, pp. 367-374, 1959.

[39] E. E. Ebert, "Fuzzy verification of high-resolution gridded forecasts: a review and proposed framework," Meteorological Applications, vol. 15, no. 1, pp. 51-64, 2008.

[40] C. Kummerow, J. Simpson, O. Thiele et al., "The status of the tropical rainfall measuring mission (TRMM) after two years in orbit," Journal of Applied Meteorology, vol. 39, no. 12, pp. 1965-1982, 2000.

[41] G. J. Huffman, D. T. Bolvin, E. J. Nelkin et al., "The TRMM multisatellite precipitation analysis (TMPA): quasi-global, multiyear, combined-sensor precipitation estimates at fine scales," Journal of Hydrometeorology, vol. 8, no. 1, pp. 38-55, 2007.

[42] C. Aybar, W. Lavado-Casimiro, A. Huerta et al., Uso del Producto Grillado "PISCO" de precipitación en Estudios, Investigaciones y Sistemas Operacionales de Monitoreo y Pronóstico Hidrometeorológico, Nota Técnica 001 SENAMHI-DHI-2017, Senamhi, Lima, Peru, 2017, ftp://ftp.senamhi.gob.pe/PISCO_ v2.0/PISCO-Prec-v2.0.pdf.

[43] S. P. Chavez and K. Takahashi, "Orographic rainfall hot spots in the Andes-Amazon transition according to the TRMM precipitation radar and in situ data," Journal of Geophysical Research: Atmospheres, vol. 122, no. 11, pp. 5870-5882, 2017. 

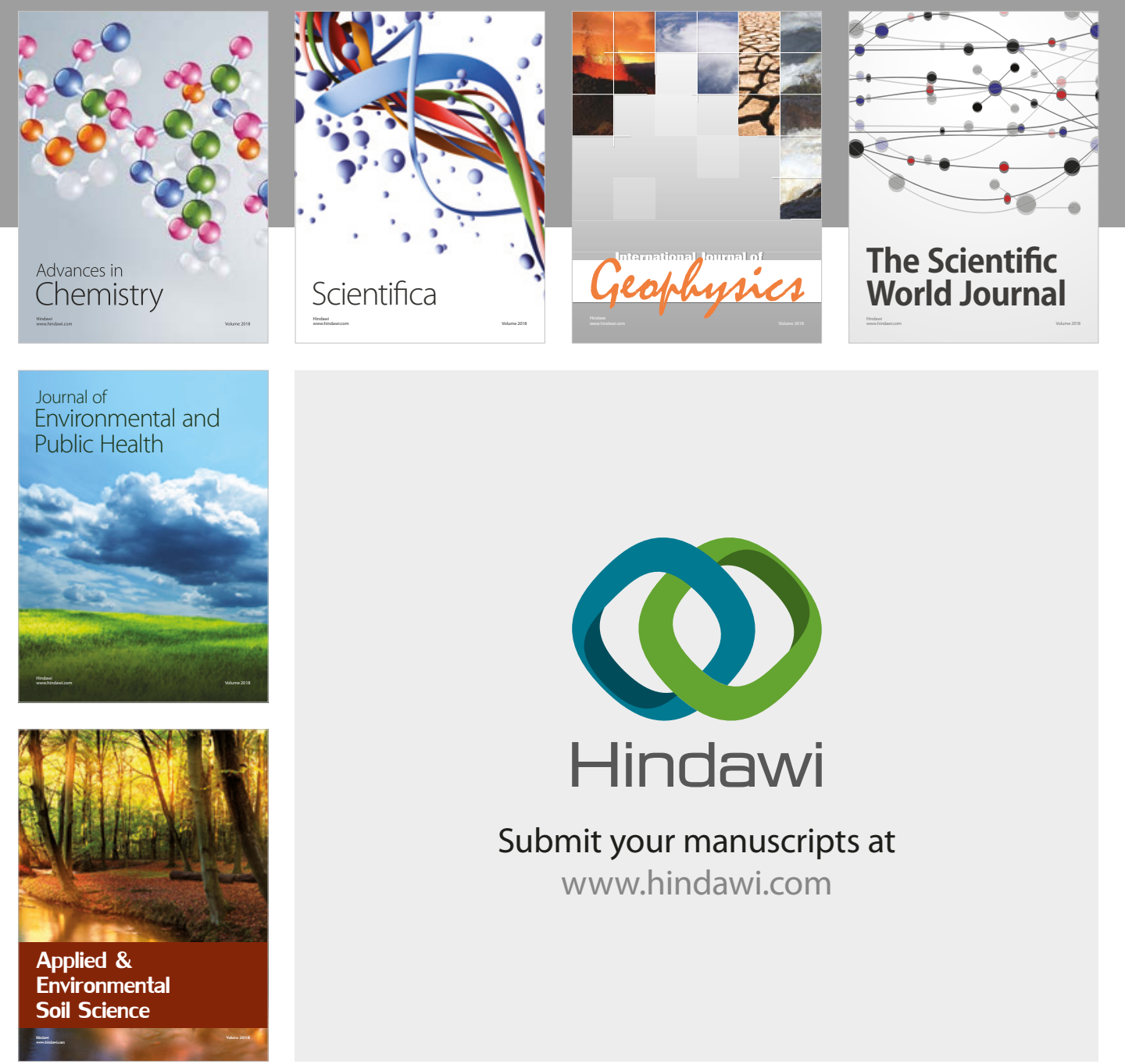

The Scientific

\section{World Journal}
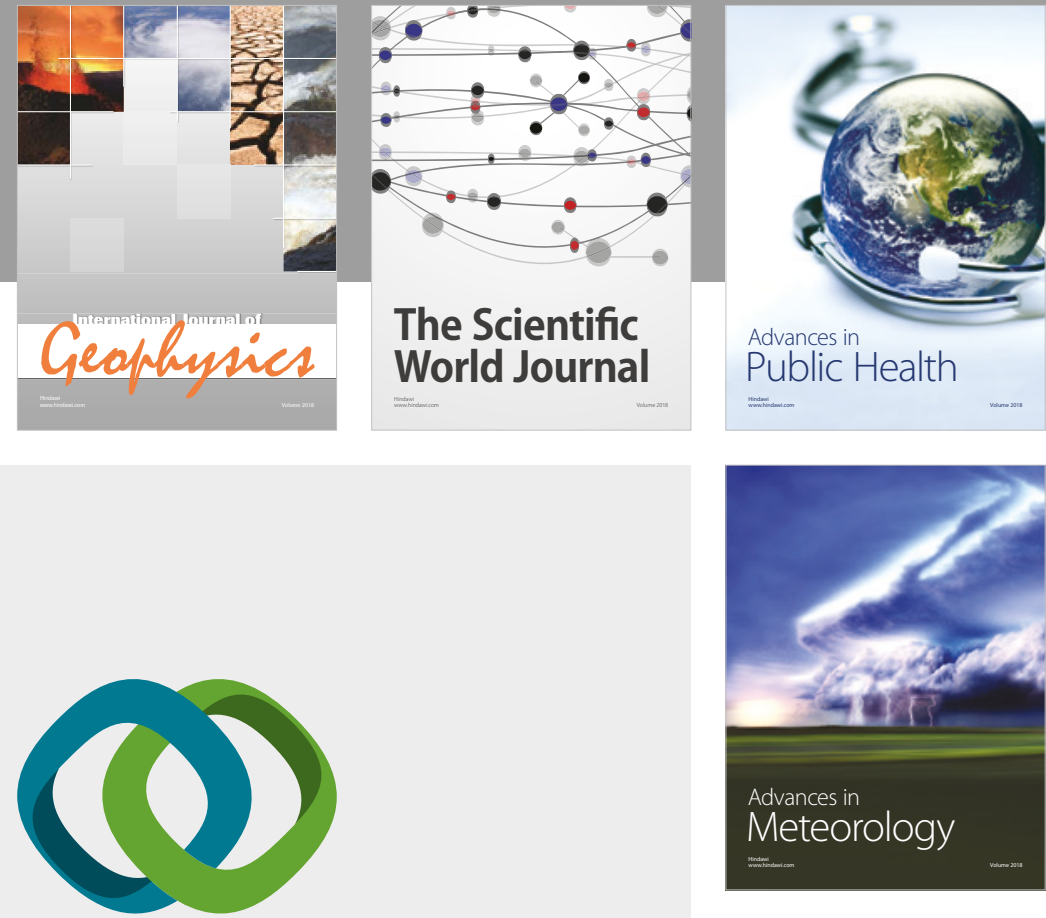

Advan

Public Health

\section{Hindawi}

Submit your manuscripts at

www.hindawi.com
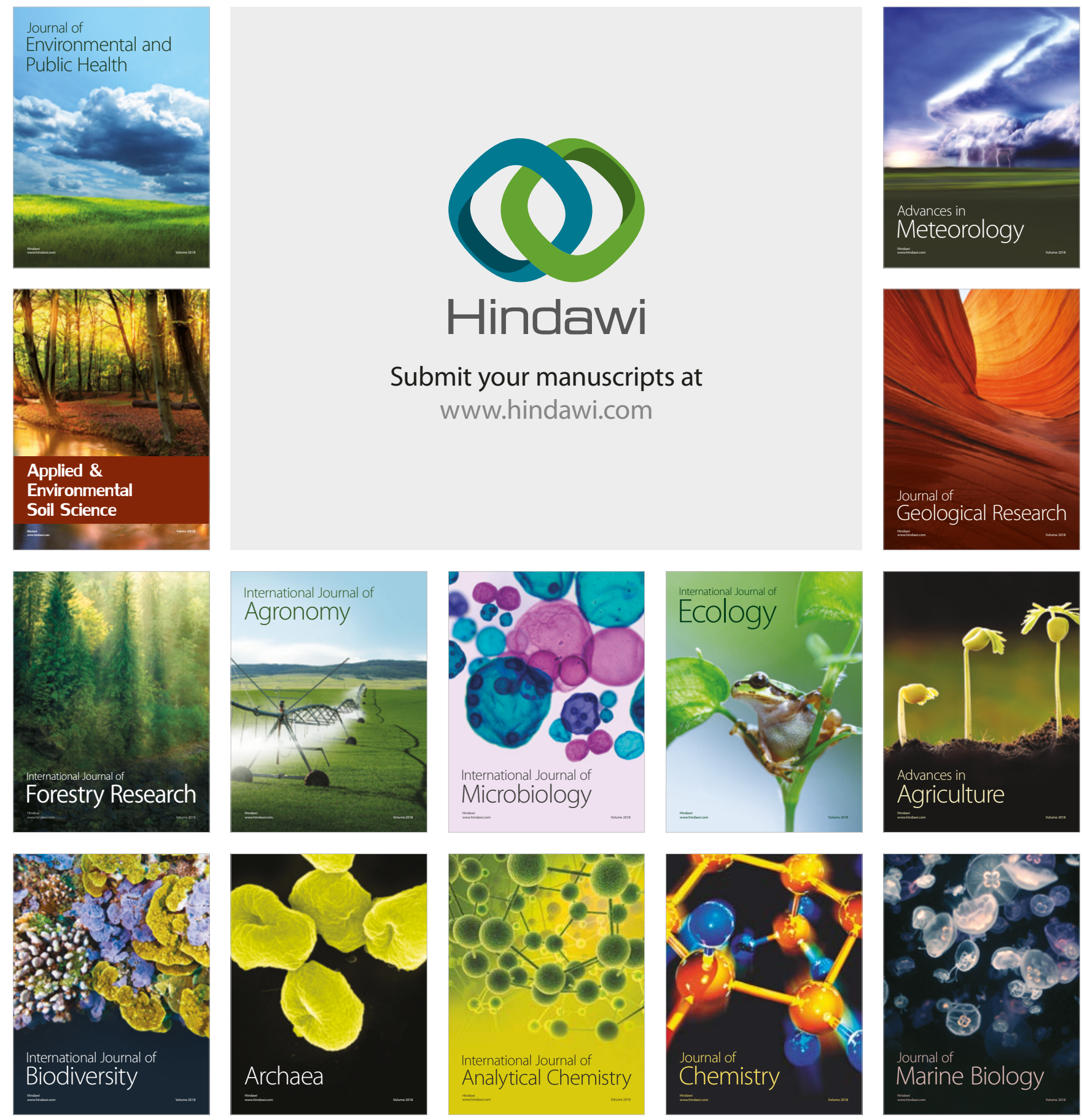